\title{
A strong version of Implicit Function Theorem
}

\author{
Genrich Belitskii ${ }^{1}$ • Dmitry Kerner ${ }^{1}$
}

Received: 15 June 2015 / Revised: 27 October 2015 / Accepted: 29 October 2015 /

Published online: 14 January 2016

(C) Springer International Publishing AG 2016

\begin{abstract}
We suggest the necessary/sufficient criteria for existence of a (order-byorder) solution $y(x)$ of a functional equation $F(x, y)=0$ over a ring. In full generality, the criteria hold in the category of filtered groups, this includes the wide class of modules over (commutative, associative) rings. The classical Implicit Function Theorem and its strengthening obtained by Tougeron and Fisher appear to be (weaker) particular forms of the general criterion. We obtain a special criterion for solvability of equations arising from group actions $g(w)=w+u$, here $u$ is "small". As an immediate application we re-derive the classical criteria of determinacy, in terms of the tangent space to the orbit. Finally, we prove the Artin-Tougeron-type approximation theorem: if a system of $C^{\infty}$-equations has a formal solution and the derivative satisfies a Lojasiewicz-type condition then the system has a $C^{\infty}$-solution.
\end{abstract}

Keywords Equations over local rings · Equations on groups · Functional equations · Tougeron's Implicit Function Theorem · Artin's approximation theorem · Tougeron's approximation theorem $\cdot$ Finite determinacy

Mathematics Subject Classification $\quad 47 \mathrm{~J} 07 \cdot 26 \mathrm{~B} 10 \cdot 30 \mathrm{D} 05 \cdot 39 \mathrm{Bxx} \cdot 65 \mathrm{Q} 20$

Dmitry Kerner was partially supported by the grant FP7-People-MCA-CIG, 334347 and the Grant ISF $844 / 14$.

$凶$ Dmitry Kerner

dmitry.kerner@gmail.com

Genrich Belitskii

genrich@math.bgu.ac.il

1 Department of Mathematics, Ben Gurion University of the Negev, P.O.B. 653, Beersheba 84105, Israel 


\section{Introduction}

All rings in this paper are commutative, associative, with unit element, of zero characteristic. We use the multivariable notation $x=\left(x_{1}, \ldots, x_{m}\right), y=\left(y_{1}, \ldots, y_{n}\right)$.

\subsection{General setting and known results}

Consider a system of (analytic/formal/ $C^{\infty} / C^{k}$ ) equations $F(x, y)=0$. The classical Implicit Function Theorem reads: If the matrix of derivatives, $\partial_{y} F(0,0)=F_{y}^{\prime}(0,0)$, is right invertible (i.e. is of the full rank) then $F(x, y)=0$ has a (analytic/formal/etc.) solution.

The condition " $F_{y}^{\prime}(0,0)$ is right invertible" is quite restrictive. For example, the theorem does not ensure a solution of the one-variable equation $x y=0$ (in the vicinity of $(0,0))$ or of $y^{2}=0$ (at any point).

Various strengthenings/generalizations of this theorem are known (including the Hensel lemma). For example, the Tougeron Implicit Function Theorem ensures solvability when the matrix $F_{y}^{\prime}(x, 0)$ is not too degenerate. Denote by $I_{\max } F_{y}^{\prime}(x, 0)$ the ideal of the maximal minors of this matrix.

Theorem 1.1 ([29], [30, p. 56]) Let $R=\mathbb{k}[[x, y]]$ or $\mathbb{k}\{x, y\}$ (for $\mathbb{k}$ a normed field) or $C^{\infty}\left(\mathbb{R}^{m} \times \mathbb{R}^{n}, 0\right)$. Let $F(x, y) \in R^{\oplus p}, p \leqslant n$, and let $I \subset R$ be a proper ideal. If $F(x, 0) \in I \cdot\left(I_{\max } F_{y}^{\prime}(x, 0)\right)^{2} R^{\oplus p}$ then there exists a solution $F(x, y(x)) \equiv 0$ such that $y(x) \in I R^{\oplus n}$.

While this theorem ensures the solution of $y x=0$ and $y^{2}=0$, it fails to ensure a solution of the system

$$
\left\{\begin{array}{l}
y_{1}^{2}+y_{1} x=x^{3} \\
y_{2}^{2}+y_{2} x=x^{3}
\end{array}\right.
$$

Here $F(x, 0)=x^{3}\left(\begin{array}{l}1 \\ 1\end{array}\right), I_{\max } F_{y}^{\prime}(x, 0)=\left(x^{2}\right)$, thus $F(x, 0) \notin\left(I_{\max } F_{y}^{\prime}(x, 0)\right)^{2}$.

It was noticed by Tougeron [28] that one can replace in the condition $F(x, 0) \in$ $I \cdot\left(I_{\max } F_{y}^{\prime}(x, 0)\right)^{2} R^{\oplus p}$ the ideal $I_{\max } F_{y}^{\prime}(x, 0)$ by a larger ideal,

$$
\mathfrak{a}_{F_{y}^{\prime}(x, 0)}=\operatorname{ann}\left(\operatorname{coker}\left(F_{y}^{\prime}(x, 0)\right)\right),
$$

the annihilator of the cokernel of the morphism $R^{\oplus n} \stackrel{F_{y}^{\prime}(x, 0)}{\longrightarrow} R^{\oplus p}$. Some properties of this ideal are recalled in Sect. 2.3. By now we just mention that for $p=1$, i.e. the case of one equation, the two ideals coincide: $\mathfrak{a}_{F_{y}^{\prime}(x, 0)}=I_{\max } F_{y}^{\prime}(x, 0)$.

The statement was further strengthened by Fisher, he replaced one of the factors in $\left(\mathfrak{a}_{F_{y}^{\prime}(x, 0)}\right)^{2}$ by the image $\operatorname{Im} F_{y}^{\prime}(x, 0) \subseteq R^{\oplus p}$. (The initial version was for $p$-adic rings, we give a more general version relevant to our context.)

Theorem 1.2 ([10]) Let $(R, \mathfrak{m})$ be a local Henselian ring over a field of zero characteristic. Let $F_{1}, \ldots, F_{p} \in R\left[\left[y_{1}, \ldots, y_{p}\right]\right]$. Suppose

$$
F(x, 0) \in \mathfrak{m} \cdot \mathfrak{a}_{F_{y}^{\prime}(x, 0)} \cdot \operatorname{Im} F_{y}^{\prime}(x, 0) .
$$


Then there exists a solution $F(x, y(x)) \equiv 0$ such that $y(x) \in \mathfrak{m} \cdot \mathfrak{a}_{F_{y}^{\prime}(x, 0)} \cdot R^{\oplus p}$.

In the case of one equation, $p=1$, this coincides with Tougeron's result. For $p>1$, Fisher's result is stronger. (Note that $\mathfrak{a}_{F_{y}^{\prime}(x, 0)} \cdot \operatorname{Im} F_{y}^{\prime}(x, 0) \supseteq\left(\mathfrak{a}_{F_{y}^{\prime}(x, 0)}\right)^{2} R^{\oplus p}$, and for $p>1$ the inclusion is in general proper.)

Though Fisher's version solves the examples mentioned above, it cannot cope with a slightly more complicated example

$$
y_{1}^{2}-y_{2}^{2}+y_{1} x_{1}^{k}+y_{2} x_{2}^{k}+g\left(x_{1}, x_{2}\right)=0,
$$

where $\mathfrak{m}=\left(x_{1}, x_{2}\right) \subset R=\mathbb{k}\left[\left[x_{1}, x_{2}\right]\right]$ and $g\left(x_{1}, x_{2}\right) \in \mathfrak{m}^{2 k+1}$ for $k>2$. Here

$$
\mathfrak{m} \cdot \mathfrak{a}_{F_{y}^{\prime}(x, 0)} \cdot \operatorname{Im} F_{y}^{\prime}(x, 0)=\mathfrak{m} \cdot\left(x_{1}^{k}, x_{2}^{k}\right)^{2} \subsetneq \mathfrak{m}^{2 k+1},
$$

thus in general $F(x, 0)=g\left(x_{1}, x_{2}\right) \notin \mathfrak{m} \cdot \mathfrak{a}_{F_{y}^{\prime}(x, 0)} \cdot \operatorname{Im} F_{y}^{\prime}(x, 0)$, i.e. condition (1) is not satisfied.

\subsection{Overview of results}

Our work has began from the observation that Fisher's condition can be further weakened: instead of $F(x, 0) \in \mathfrak{m} \cdot \mathfrak{a}_{F_{y}^{\prime}(x, 0)} \cdot \operatorname{Im} F_{y}^{\prime}(x, 0)$ it is enough to ask for $F(x, 0) \in \mathfrak{m}$. $J \cdot \operatorname{Im} F_{y}^{\prime}(x, 0)$, where $\mathfrak{a}_{F_{y}^{\prime}(x, 0)} \subseteq J \subset R$ is the biggest possible ideal satisfying $J^{2}=J \cdot \mathfrak{a}_{F_{y}^{\prime}(x, 0)}$. (See Corollary 3.17 and Sect. 4.1 for more detail.) This gives further strengthening of Fisher's and Tougeron's statements. Still, this strengthening does not help to address the very simple system (cf. Sect. 4.1)

$$
\left\{\begin{array}{l}
y_{1}^{2}+y_{1} x_{1}=x_{1}^{100} \\
y_{2}^{2}+y_{2} x_{2}=x_{2}^{100}
\end{array}\right.
$$

Here $\mathfrak{a}_{F_{y}^{\prime}}=\left(x_{1} x_{2}\right)$ thus $J=\left(x_{1} x_{2}\right)$, but $F(x, 0) \notin J \cdot \operatorname{Im} F_{y}^{\prime}(x, 0)$.

In this note we prove much stronger solvability criteria. In this introduction we sketch just the main features of the method. The detailed formulation can be found in Sect. 3.1 (Theorem 2.1) and Sect. 5 (Theorem 5.3), the applications are in Sects. 4 and 6.

We weaken the condition on $F_{y}^{\prime}(x, 0)$ further, to the "weakest possible" condition of "iff" type, so that we get a Strong Implicit Function Theorem.

Our results hold in broader category. It is natural to extend from the classical case of $\mathbb{k}[[x, y]], \mathbb{k}\{x, y\}, C^{p}\left(\mathbb{R}_{x}^{m} \times \mathbb{R}_{y}^{n}, 0\right)$ to the local Henselian rings (not necessarily regular or Noetherian) over a field. In fact even the ring structure is not necessary, our main result, Theorem 2.1, is true for filtered (not necessarily abelian) groups.

A particular class of equations comes from the group actions $G \circlearrowright W$. Assume $W$ is a filtered abelian group (e.g. a module over a local ring). To understand how large the orbit is one studies the equation $g(w)=w+u$. Here $g \in G$ is an unknown, while $u \in W$ is "small". (More precisely, one studies whether the orbit $G w$ is open in the 
topology defined by a filtration.) Theorem 2.1, being very general, is of little use here. Rather, we obtain a special version of strong IFT, Sect. 3.1.2.

Usually the main problem is to establish the order-by-order solution procedure. Thus many of our results are of the form: If (...) then there exists a Cauchy sequence $\left\{y^{(n)}(x)\right\}_{n}$ such that $F\left(x, y^{(n)}(x)\right) \rightarrow 0$. (The topology here is induced by a filtration, e.g. by the powers of maximal ideal.)

Once such a result is established, one has a solution in the completion of $R^{\oplus p}$ by the filtration. Then (if $R$ is non-complete) one uses the Artin-type approximation theorems [18] to establish a solution over $R$, or at least over the henselization of $R$.

For the ring $C^{\infty}\left(\mathbb{R}^{p}, 0\right)$ and many other important rings the Artin approximation does not hold (in the naive way). Over some rings we can directly ensure a solution, see Sect. 3.4. For $C^{\infty}\left(\mathbb{R}^{p}, 0\right)$ we use Theorem 5.3.

\subsection{Comments and motivation}

Several remarks/explanations are necessary at this point. Recall the simple geometric interpretation. Consider the (germ of) subscheme/subspace $\{F(x, y)=0\} \subset$ $(X, 0) \times(Y, 0)$. The classical IFT, in the case when $(X, 0)$ and $(Y, 0)$ are smooth, gives a sufficient condition that the germ $\{F(x, y)=0\}$ is smooth and its projection onto $(X, 0)$ is an isomorphism.

Our version of IFT, for arbitrary Henselian germs $(X, 0),(Y, 0)$, gives a necessary and sufficient condition that the germ $\{F(x, y)=0\}$ has an irreducible component whose projection onto $(X, 0)$ is an isomorphism. This can be restated as follows. Consider the natural projection $\{F(x, y)=0\} \stackrel{\pi}{\longrightarrow}(X, 0)$. Usually this projection is not an isomorphism. The solvability of the equation means the weaker property: the existence of the section of $\pi,(X, 0) \stackrel{s}{\rightarrow}\{F(x, y)=0\}$.

To emphasize: as the germ $\{F(x, y)=0\}$ is in general non-smooth (possibly reducible, non-reduced), the question cannot be simply "linearized" by an automorphism of $(X, 0) \times(Y, 0)$, i.e. cannot be reduced to the classical IFT by some appropriate change of variables.

A reformulation in terms of commutative algebra reads: Given a ring $R$ over some base ring $R_{X}$, e.g. $R=R_{X}[y]$ or $R=R_{X}[[y]]$, etc. Given an ideal $F=$ $\left(F_{1}, \ldots, F_{p}\right) \subset R$, a solution of $F(x, y)=0$ is a projection $R \stackrel{y \rightarrow y(x)}{\longrightarrow} R_{X}$ whose kernel is precisely $F$.

The classical approach to construct a solution is the order-by-order approximation: first solve the part linear in $y$ (modulo quadratic terms), then quadratic, cubic, etc. Accordingly, we always present the equation(s) $F(x, y)=0$ in the form $u+L y+$ $H(y)=0 \in W$. Here $u=F(x, 0) \in W, V \stackrel{L=F_{y}^{\prime}(x, 0)}{\longrightarrow} W$ is a homomorphism of $R$ modules (or just of abelian groups); $H(y)$ denotes the remaining "higher order terms" (a contractive map in the sense of Krull topology), defined in Sect. 2.2.

Further, as we always start from a solution of the linear part, $u+L y=0$, we assume $u \in L(V)$, i.e. $u=-L v$ for some $v \in V$. Therefore the equation to solve is presented in the form

$$
L(y-v)+H(y)=0 .
$$


In practice one usually needs not just $a$ solution. Thinking of $v$ as a parameter, one needs a statement of the type:

There exists a subgroup/submodule $V_{1} \subseteq V$ such that for any $v \in V_{1}$ the equation $L(y-v)+H(y)=0$ has a solution $y_{v} \in V_{1}$ which is "close" to $v$ and depends on $v$ "differentiably". We call this a good solution, the precise formulation see in Sect. 2.2. Our criteria answer the question:

Question 1.3 Given $V \stackrel{L, H}{\longrightarrow} W$, what is the biggest $V_{1} \subseteq V$ such that for any $v \in V_{1}$ there exists a good solution?

Note that for some equations all solutions are "not good", cf. Sect. 4.3.

If the number of unknowns equals the number of equations and $F_{y}^{\prime}(0,0)$ is nondegenerate, then the classical IFT ensures the unique solution. When $F_{y}^{\prime}(0,0)$ is degenerate the solution (if it exists) can be non-unique, as the space $\{F(x, y)=0\}$ can have several irreducible components. However, when $L$ is injective, the solution lying in $V_{1}$ is unique! The (non-)uniqueness issues are addressed in Sect. 3.3.

We expand $F(x, y)=0$ in powers of $y$ (i.e. at $y=0$ ), hence the criteria are formulated in terms of $F(x, 0), F_{y}^{\prime}(x, 0)$, etc. One can expand at some other point, $y=y^{(0)}(x)$, then the criteria are written in terms of $F\left(x, y^{(0)}(x)\right), F_{y(0)}^{\prime}(x)\left(x, y^{(0)}(x)\right)$, etc. (For example, Theorem 1.2 is stated in [10] in such a form.) Such an expansion at $y^{(0)}(x)$ is helpful if one has a good initial approximation for the solution. The two approaches are obviously equivalent, e.g. by changing the variable $y \mapsto y-y^{(0)}(x)$. To avoid cumbersome formulas we always expand at $y=0$.

In view of our initial result, Sect. 1.2, one might try to weaken the condition on the ideal $\mathfrak{a}_{F_{y}^{\prime}(x, 0)} \subseteq J \subset R$ further. It appears that $J^{2}=J \mathfrak{a}_{F_{y}^{\prime}(x, 0)}$ is almost the "weakest possible" among the conditions stated in terms of ideals only, it cannot be significantly weakened, cf. Sect. 4.2. But this condition is still far from being necessary. The "right" condition (necessary and sufficient) is obtained by replacing the ideals with filtered subgroups. As a bonus we do not need the rings structure anymore, e.g. Theorem 2.1 holds in the generality of (not necessarily abelian) filtered groups.

If the equations $F(x, y)=0$ are linear in $y$, i.e. $F(x, y)=F(x, 0)+F_{y}^{\prime}(x, 0) y$, then the obvious sufficient condition for solvability is: the entries of $F(x, 0)$ lie in the ideal $\mathfrak{a}_{F_{y}^{\prime}(x, 0)}$. While the (tautological) necessary and sufficient condition is: $F(x, 0) \in$ $\operatorname{Im} F_{y}^{\prime}(x, 0)$. This condition is much weaker than those of Tougeron and Fisher and is far from being sufficient for non-linear equations. Therefore as landmarks for our criteria one should consider equations that are non-linear in $y$.

The Implicit Function Theorem is a fundamental result. In Sect. 4.4 we obtain an immediate corollary to non-bifurcation of multiple polynomial roots under deformations. In Sect. 4.5 we indicate a potential application to the study of smooth curve-germs (lines/arcs) on singular spaces. In Sect. 6.3 we apply a version of strong IFT to groupactions to re-derive the classical criteria of finite determinacy.

Further directions in algebra and geometry are: matrix equations, equations on (filtered) groups [3], tactile maps [6], bounds on Artin-Greenberg functions [23,24], etc. We hope to report on these applications soon. 


\section{Definitions and notations}

\subsection{Groups with descending filtration}

We always assume that a (not necessarily abelian) group $V$ is filtered by a sequence of normal subgroups $V \supset V_{1} \supset V_{2} \supset \cdots, V_{j} \triangleleft V$. Moreover, we assume that the filtration satisfies the condition $\left[V_{1}, V_{i}\right] \subseteq V_{i+1}$, similarly to the lower central series of a group. This later condition is trivial when $V$ is an abelian group. If $V$ is complete with respect to $\left\{V_{i}\right\}$ then the filtration is faithful, i.e. $\bigcap_{i \geqslant 1} V_{i}=\left\{\mathbb{1}_{V}\right\}$. The filtration induces the Krull topology, the fundamental system of neighborhoods of $v \in V$ is $\left\{v V_{j}\right\}_{j \geqslant 1}$, or $\left\{V_{j} v\right\}_{j} \geqslant 1$, by normality.

Example 2.1 (a) The simplest case is when $V$ is a module over a ring, with filtration defined by the powers of an ideal $V_{j}=I^{j} V$.

(b) Let $(R, \mathfrak{m})$ be a local ring with the filtration $R \supset \mathfrak{m} \supset \mathfrak{m}^{2} \supset \cdots$. Consider the group of invertible $R$-matrices $V=\operatorname{GL}(n, R)$. We get the filtration by normal subgroups $V_{j}=\left\{\mathbb{1}+A: A \in \operatorname{Mat}\left(n, n ; \mathfrak{m}^{j}\right)\right\}$.

(c) Let $(X, 0)$ be the germ of a space (algebraic/formal/analytic etc). Consider the group of its automorphisms $V=\operatorname{Aut}(X, 0)$. The natural filtration is by the subgroups of automorphisms that are identity up to $j$ 'th order. More precisely, denote by $\left(R_{(X, 0)}, \mathfrak{m}\right)$ the local ring of (germs of) regular functions. Then

$$
\operatorname{Aut}^{(j)}(X, 0)=\left\{\phi \circlearrowright R_{(X, 0)}: \phi(f)-f \in \mathfrak{m}^{j+1}, f \in R\right\}
$$

\subsection{Implicit function equation}

Given two (not necessarily abelian) groups $V, W$, a homomorphism $V \stackrel{L}{\longrightarrow} W$, and a decreasing filtration $\left\{V_{j}\right\}$ by normal subgroups, we define the filtration $W_{j}=L\left(V_{j}\right)$ on $W$. Consider the equation $L(y) H(y)=L(v)$, where the "higher order" map $V \stackrel{H}{\longrightarrow} W$, usually not a homomorphism, is such that

- $H\left(\mathbb{1}_{V}\right)=\mathbb{1}_{W}$,

- $(H(y))^{-1} H\left(y V_{j}\right) \subseteq L\left(V_{j+1}\right)$ for any $y \in V_{1}$ and $j \in \mathbb{N}$.

Note that being of higher order depends essentially on $L$, in particular, $H\left(V_{j}\right) \subseteq$ $L\left(V_{j+1}\right)$.

If $V, W$ are abelian groups, then the implicit function equation is $L(y-v)+H(y)=$ 0 , where $L \in \operatorname{Hom}(V, W)$, while the higher order $H(y)$ satisfies $H\left(0_{V}\right)=0_{W}$ and $H\left(y+V_{j}\right)-H(y) \subseteq L\left(V_{j+1}\right)$ for any $y \in V_{1}$ and $j$.

The most common case is when $V, W$ are modules over a (commutative, associative) ring $R$. Then usually $L \in \operatorname{Hom}_{R}(V, W)$. We say that the map $V \stackrel{H}{\longrightarrow} W$ is of order $\geqslant k$ if for any ideal $J \subset R$ there holds $H(J V) \subseteq J^{k} W$.

Example 2.2 Suppose $R$ is graded, fix an ideal $J \subset R$ and consider the filtration $V_{j}=J^{j} V$. Suppose $H(y)$ can be written as a sum of homogeneous forms $H(y)=$ $\sum_{i \geqslant k} h_{i}(y)$, the degree of $h_{i}(y)$ being $i$. If $L(V) \supseteq J^{k-2} W$ then $H(y)$ is a "higher 
order" term for $L$. Indeed, for any $i \geqslant k$ and $y \in J V$,

$$
h_{i}\left(y+V_{j}\right)-h_{i}(y) \in J^{i-1} \cdot J^{j} W \subseteq J^{k-1+j} W \subseteq J^{j+1} L(V) .
$$

Example 2.3 (Warning) Being of higher order terms can be a restrictive condition. For example, in the equation $y^{2}-y x+x^{a}=0$ the monomial $y^{2}$ represents the higher order term for the filtration $V_{j}=\left(x^{j}\right)$ only if $a \geqslant 3$. Otherwise the condition $H\left(V_{1}\right) \subseteq L\left(V_{2}\right)$ is not satisfied.

An order-by-order solution of the equation $L(y) H(y)=L(v)$ is a Cauchy sequence $\left\{y^{(n)}\right\}_{n} \geqslant 1$ with respect to the filtration $V_{\bullet}$, i.e. $y^{(n)}\left(y^{(n+1)}\right)^{-1} \in V_{n}$, such that $L\left(y^{(n)}\right)$ $H\left(y^{(n)}\right) L(v)^{-1} \in L\left(V_{n}\right)$. By normality $V_{n} \triangleleft V$, we can also write the condition as $\left(y^{(n+1)}\right)^{-1} y^{(n)} \in V_{n}$ or $L(v)^{-1} L\left(y^{(n)}\right) H\left(y^{(n)}\right) \in L\left(V_{n}\right)$.

We say that the equation $L(y) H(y)=L(v)$ admits a good solution on $V_{1}$ if there exists a map $V_{1} \stackrel{y}{\longrightarrow} V_{1}$ satisfying the following conditions (we denote $y(v)$ by $y_{v}$, i.e. consider $v$ as a parameter):

- $L\left(y_{v}\right) H\left(y_{v}\right)=L(v)$ for any $v \in V_{1}$;

- $y_{\mathbb{1}_{V}}=\mathbb{1}_{V}$ and $y$ respects the filtration, $y\left(V_{i}\right) \subseteq V_{i}$ (this is a strengthening of continuity);

- the map $y$ is "differentiable and close to identity", namely, $y_{v}=g(v) \cdot v$, where the map $V_{1} \stackrel{g}{\rightarrow} V_{1}$ is such that $g\left(v \cdot V_{j}\right) g^{-1}(v) \in V_{j+1}$ for any $v \in V_{1}$ and $j \in \mathbb{N}$. Alternatively this condition can be stated as $y_{v \Delta_{j}} \Delta_{j}^{-1} y_{v}^{-1} \in V_{j+1}$ for any $\Delta_{j} \in V_{j}$. By normality, this is equivalent to $\Delta_{j}^{-1} y_{v}^{-1} y_{v \Delta_{j}} \in V_{j+1}$.

We say that a solution $V_{1} \stackrel{y}{\longrightarrow} V_{1}$ is quasi-good if $y_{v}=g(v) \cdot v$, where $g\left(V_{j}\right) \subseteq V_{j+1}$. Good implies quasi-good.

Combining these notions, we get the notion of a good order-by-order solution, i.e. a Cauchy sequence of maps $\left\{V_{1} \stackrel{y^{(n)}}{\longrightarrow} V_{1}\right\}_{n}$ with

- $y_{\mathbb{1}_{V}}^{(n)}=\mathbb{1}_{V}, L\left(y_{v}^{(n)}\right) H\left(y_{v}^{(n)}\right) L(v)^{-1} \in L\left(V_{n}\right)$,

- $y_{v \Delta_{j}}^{(n)} \Delta_{j}^{-1}\left(y_{v}^{(n)}\right)^{-1} \in V_{j+1}$ for all $n, j \geqslant 1$ and $\Delta_{j} \in V_{j}$.

Similarly, a quasi-good order-by-order solution satisfies $y_{v}^{(n)}=g^{(n)}(v) \cdot v$, where $g^{(n)}\left(V_{j}\right) \subseteq V_{j+1}$.

If $V, W$ are abelian groups then all notions simplify accordingly. A good orderby-order solution means a Cauchy sequence of maps $\left\{V_{1} \stackrel{y^{(n)}}{\longrightarrow} V_{1}\right\}_{n}$ satisfying the conditions:

- $y_{v}^{(n)}-y_{v}^{(n+1)} \in V_{n}, L\left(y_{v}^{(n)}-v\right)+H\left(y_{v}^{(n)}\right) \in L\left(V_{n}\right)$,

- $y_{v+\Delta_{j}}^{(n)}-y_{v}^{(n)}-\Delta_{j} \in V_{j+1}$ for all $n, j \geqslant 1$ and $\Delta_{j} \in V_{j}$.

\subsection{Annihilator of cokernel}

Consider a homomorphism of finitely generated $R$-modules $V \stackrel{L}{\longrightarrow} W$. Its image $L(V)$ is an $R$-submodule of $W$. Its cokernel $\operatorname{coker}(L)=W / L(V)$ is an $R$-module as well. The annihilator-of-cokernel ideal is defined as the support of the cokernel module

$$
\mathfrak{a}_{L}=\operatorname{ann}(\operatorname{coker}(L))=\{f \in R: f W / L(V)=\{0\} \text {, i.e. } f W \subseteq L(V)\} .
$$


Recall the classical relation [9, Proposition 20.7]: for $L \in \operatorname{Mat}(m, n ; R)$ with $m \leqslant n$ there holds $\mathfrak{a}_{L} \supseteq I_{m}(L) \supseteq\left(\mathfrak{a}_{L}\right)^{m}$. (Here $I_{m}(L)=I_{\max }(L)$ is the ideal of the maximal, i.e. $m \times m$, minors.) In particular, for $m=1, \mathfrak{a}_{L}=I_{1}(L)$, the ideal is generated by all entries of $L$.

By definition, $\mathfrak{a}_{L} W \subseteq L(V)$. In many cases one has a stronger property: $\mathfrak{a}_{L} W \subseteq$ $J L(V)$ for some proper ideal $J \subsetneq R$.

Example 2.4 Let $L \in \operatorname{Mat}(m, n ; J), 1<m \leqslant n$, and suppose $\mathfrak{a}_{L}=I_{\max }(L)$, e.g. this holds when $I_{\max }(L)$ is radical. Then $\mathfrak{a}_{L} W \subseteq J^{m-1} L(V)$.

The embedding $\mathfrak{a}_{L} W \subseteq \mathfrak{m} L(V)$ does not hold only in some degenerate cases. For example, let $L=\left(\begin{array}{cc}f & 0 \\ 0 & L_{1}\end{array}\right)$, where $\operatorname{det} L_{1}=f$. Then $\mathfrak{a}_{L}=(f)$ and $(f) W \nsubseteq \mathfrak{m} \cdot L(V)$.

\section{Main results: criteria of solvability}

\subsection{General statements}

Let $V \stackrel{L}{\longrightarrow} W$ be a homorphism of (arbitrary) groups, where $V$ is filtered by normal subgroups as in Sect. 2.1. Consider the equation $L(y) H(y)=L(v)$. See Sect. 2.2 for definitions.

Theorem 2.1 (i) If the map $V \stackrel{H}{\longrightarrow} W$ represents the "higher order terms", i.e. $H(y)^{-1} H\left(y V_{j}\right) \subseteq L\left(V_{j+1}\right)$ for any $y \in V_{1}, j \in \mathbb{N}$, then there exists a quasigood order-by-order solution $V_{1} \stackrel{y^{(n)}}{\longrightarrow} V_{1}$. If moreover $L$ admits a right inverse, i.e. there exists a map $L(V) \stackrel{T}{\longrightarrow} V$ such that $L \circ T=\mathbb{1}_{L(V)}, T\left(L\left(V_{i}\right)\right) \subseteq V_{i}$, then there exists a good order-by-order solution.

(ii) Suppose $V \stackrel{H}{\longrightarrow} W$ is compatible with the filtration in the sense $H\left(y \cdot V_{j}\right) \subseteq$ $H(y) \cdot L\left(V_{N(j)}\right)$ for some $N(j), \lim _{j \rightarrow \infty} N(j)=\infty$. If there exists a goodorderby-order solution $V_{1} \stackrel{y^{(n)}}{\longrightarrow} V_{1}$, then $H$ represents the "higher order terms", i.e. $H\left(y V_{j}\right) \subseteq H(y) \cdot L\left(V_{j+1}\right)$ for any $y \in V_{1}, j \in \mathbb{N}$.

(iii) If $V$ is complete with respect to $V_{\bullet}$ and $H$ represents the "higher order terms" then there exists a quasi-good solution $V_{1} \stackrel{y}{\longrightarrow} V_{1}$. If moreover $L$ admits a right inverse, then there exists a good solution.

Proof (i) First we construct a quasi-good order-by-order solution $y^{(n)}$. The procedure is inductive with non-canonical choices. If $L$ is right invertible then all choices are canonical and the solution becomes good.

Note that $H\left(V_{i}\right) \subseteq L\left(V_{i+1}\right)$, cf. Sect. 2.2. Fix some $v \in V_{i}$, we construct inductively $y^{(n)}$ such that $y^{(n+1)}\left(y^{(n)}\right)^{-1} \in V_{i+n}$ and $L\left(y^{(n)}\right) H\left(y^{(n)}\right) L(v)^{-1} \in L\left(V_{i+n}\right)$.

Choose $y^{(1)}=v$ and note that $L\left(y^{(1)}\right) H\left(y^{(1)}\right) L\left(v^{-1}\right)=L(v) H(v) L(v)^{-1} \in$ $H\left(V_{i}\right) \subseteq L\left(V_{i+1}\right)$. Suppose $y^{(1)}, \ldots, y^{(n)}$ have been constructed for some $n \geqslant 1$. We shall look for $y^{(n+1)}$ in the form $y^{(n+1)}=z y^{(n)}$, so we should find the necessary $z \in V_{i+n}$. Note 


$$
\begin{aligned}
& L\left(y^{(n+1)}\right) H\left(y^{(n+1)}\right) L\left(v^{-1}\right)=L(z) L\left(y^{(n)}\right) H\left(y^{(n)}\right) \\
& \cdot H\left(y^{(n)}\right)^{-1} H\left(z y^{(n)}\right) L(v)^{-1} \\
& =(L(z) \underbrace{L\left(y^{(n)}\right) H\left(y^{(n)}\right) L(v)^{-1}}_{w} L(v) H\left(y^{(n)}\right)^{-1} H\left(z y^{(n)}\right) L(v)^{-1} .
\end{aligned}
$$

By the induction assumption $w \in L\left(V_{i+n}\right)$, thus we choose $z \in V_{i+n}$ such that $L(z)=w^{-1}$. (If $w$ is the identity element of $W$ then we choose $z=\mathbb{1}_{V}$.) Then equation (3) reads

$$
L\left(y^{(n+1)}\right) H\left(y^{(n+1)}\right) L\left(v^{-1}\right)=L(v) \underbrace{H\left(y^{(n)}\right)^{-1} H\left(z y^{(n)}\right)}_{\in L\left(V_{i+n+1}\right)} L\left(v^{-1}\right) \in L\left(V_{i+n+1}\right) .
$$

This completes the induction step. (Here we use the normality $V_{j} \triangleleft V$.)

By construction, $y^{(n)}$ is a Cauchy sequence, as $y^{(n)}\left(y^{(n)}\right)^{-1} \in V_{i+n}$. And if $v=\mathbb{1}_{V}$ then $y^{(n)}=\mathbb{1}_{V}$. Moreover, if $v \in V_{i}$ then $y_{v}^{(n)} v^{-1} \in V_{i+1}$. Thus $y^{(n)}$ is a quasi-good order-by-order solution.

Suppose there exists a continuous right inverse $T, L \circ T=\mathbb{1}_{L(V)}$, then in equation (3) we choose $z_{v}=T\left(L(v) H\left(y_{v}^{(n)}\right)^{-1} L\left(y_{v}^{(n)}\right)^{-1}\right)$. The proof of $y_{v \Delta_{j}}^{(n)}\left(y_{v}^{(n)} \Delta_{j}\right)^{-1} \in$ $V_{j+1}$ goes by induction on $n$. For $y_{v}^{(1)}=v$ the statement is trivial. Suppose this holds for $y_{v}^{(n)}$. Then

$$
y_{v \Delta_{j}}^{(n+1)}\left(y_{v}^{(n+1)} \Delta_{j}\right)^{-1}=\left(z_{v \Delta_{j}}^{(n+1)} y_{v \Delta_{j}}^{(n)}\right)\left(z_{v}^{(n+1)} y_{v}^{(n)} \Delta_{j}\right)^{-1}
$$

Note that $y_{v \Delta_{j}}^{(n)}\left(y_{v}^{(n)} \Delta_{j}\right)^{-1} \in V_{j+1}$, thus

$$
\left(z_{v \Delta_{j}}^{(n+1)} y_{v \Delta_{j}}^{(n)}\right)\left(z_{v}^{(n+1)} y_{v}^{(n)} \Delta_{j}\right)^{-1} \in z_{v \Delta_{j}}^{(n+1)} V_{j+1}\left(z_{v}^{(n+1)}\right)^{-1}
$$

Now, by normality $V_{j+1} \triangleleft V$, we have

$$
\begin{aligned}
z_{v \Delta_{j}}^{(n+1)} V_{j+1}\left(z_{v}^{(n+1)}\right)^{-1}= & T\left(L\left(v \Delta_{j}\right) H\left(y_{v \Delta_{j}}^{(n)}\right)^{-1} L\left(y_{v \Delta_{j}}^{(n)}\right)^{-1}\right) \\
& \cdot T\left(L(v) H\left(y_{v}^{(n)}\right)^{-1} L\left(y_{v}^{(n)}\right)^{-1}\right)^{-1} V_{j+1},
\end{aligned}
$$

which, by normality $V_{1} \triangleleft V$, equals

$$
T(L\left(\Delta_{j}\right) \underbrace{H\left(y_{v \Delta_{j}}^{(n)}\right)^{-1}}_{\in H\left(y_{v}^{(n)}\right)^{-1} V_{j+1}} L\left(y_{v \Delta_{j}}^{(n)}\right)^{-1} L\left(y_{v}^{(n)}\right) H\left(y_{v}^{(n)}\right)) V_{j+1} .
$$

Finally, by induction, this space is $T\left(L\left(\Delta_{j}\right) H\left(y_{v}^{(n)}\right)^{-1} L\left(\Delta_{j}\right)^{-1} H\left(y_{v}^{(n)}\right)\right) V_{j+1}$, and, by the property of filtration, $\left[V_{1}, V_{j}\right] \subseteq V_{j+1}$, the last expression is $V_{j+1}$.

(ii) We proceed in two steps. In Step 1 we prove that a good order-by-order solution is an almost surjective map, its image is dense. In Step 2 we use this auxiliary statement to bound $H^{-1}(y) H\left(y \Delta_{j}\right)$. 
Step 1. We prove the following auxiliary statement: If $V_{1} \stackrel{y}{\rightarrow} V_{1}$ is a good map, i.e. $y_{v}=v g(v)$ with $g\left(v V_{j}\right) g^{-1}(v) \subseteq V_{j+1}$, then the image of $y$ is dense in $V_{1}$, i.e. for any $v \in V_{1}$ there exists a sequence $v^{(n)} \in V_{1}$ such that $y_{v^{(n)}} v^{-1} \in V_{n+1}$.

Define $v^{(1)}=v$. Suppose $v^{(1)}, \ldots, v^{(i)}$ have been constructed, so that $y_{v^{(i)}} v^{-1} \in$ $V_{i+1}$, i.e. $y_{v^{(i)}} v^{-1}=\Delta_{i+1}$. Define $v^{(i+1)}=\Delta_{i+1}^{-1} v^{(i)}$. Now the direct check gives

$$
\begin{aligned}
y_{v^{(i+1)}}=y_{\Delta_{i+1}^{-1} v^{(i)}}=\Delta_{i+1}^{-1} v^{(i)} \cdot g\left(\Delta_{i+1}^{-1} v^{(i)}\right) & \in \Delta_{i+1}^{-1} v^{(i)} \cdot g\left(v^{(i)}\right) \cdot V_{i+2} \\
& =\Delta_{i+1}^{-1} y_{v^{(i)}} \cdot V_{i+2}=v \cdot V_{i+2}
\end{aligned}
$$

i.e. $v^{(i+1)}$ satisfies the needed condition.

Step 2. Fix some good order-by-order solution $V_{1} \stackrel{y^{(n)}}{\longrightarrow} V_{1}, L\left(y_{v}^{(n)}\right) H\left(y_{v}^{(n)}\right) \in$ $L(v) L\left(V_{n+1}\right)$. We should bound $H^{-1}(y) H\left(y \Delta_{j}\right)$ for any $y \in V_{1}, \Delta_{j} \in V_{j}$. By (a) we can assume $L(y) \in L\left(y_{v}^{(n)} V_{n+1}\right)$ for some $v \in V_{1}$ and $n>j$. Moreover, we can choose $n$ so large that in addition $L\left(y \Delta_{j}\right) \in L\left(y_{v \widetilde{\Delta_{j}}}^{(n)} V_{n+1}\right)$ for some $\widetilde{\Delta}_{j} \in V_{j}$. Now use $H\left(y V_{j}\right) \subseteq H(y) L\left(V_{N(j)}\right)$ and choose $n$ so large that $H(y) \in H\left(y_{v}^{(n)}\right) L\left(V_{j+1}\right)$ and $H\left(y \Delta_{j}\right) \in H\left(y_{v \widetilde{\Delta_{j}}}^{(n)}\right) L\left(V_{j+1}\right)$. Therefore, for $n>j$,

$$
\begin{aligned}
H^{-1}(y) H\left(y \Delta_{j}\right) \in & H^{-1}\left(y_{v}^{(n)}\right) H\left(y_{v \widetilde{\Delta_{j}}}^{(n)}\right) L\left(V_{j+1}\right) \\
& =\left(L\left(y_{v}^{(n)}\right)^{-1} L(v)\right)^{-1}\left(L\left(y_{v \widetilde{\Delta_{j}}}^{(n)}\right)^{-1} L\left(v \widetilde{\Delta_{j}}\right)\right) L\left(V_{j+1}\right) \\
& =L\left(v^{-1} y_{v}^{(n)}\left(y_{v \widetilde{\Delta_{j}}}^{(n)}\right)^{-1} v \widetilde{\Delta_{j}}\right) L\left(V_{j+1}\right) \\
& =L\left(g(v) g\left(v \widetilde{\Delta_{j}}\right)^{-1}\right) L\left(V_{j+1}\right) \subseteq L\left(V_{j+1}\right) .
\end{aligned}
$$

In the second row we used the goodness of $y_{v}^{(n)}$.

(iii) If $V$ is complete with respect to $V_{\bullet}$ then $\bigcap_{i \geqslant 1} V_{i}=\left\{\mathbb{1}_{V}\right\}$. Given the Cauchy sequence $y^{(n)}$ from (i), take the limit $y=\lim _{n \rightarrow \infty} y^{(n)} \in V$. Then one has

$$
L(y) H(y) L(v)^{-1}=\lim _{n \rightarrow \infty} L\left(y^{(n)}\right) H\left(y^{(n)}\right) L(v)^{-1} \in \bigcap_{n} L\left(V_{n}\right)=\left\{\mathbb{1}_{W}\right\} .
$$

Remark 2.2 To emphasize, this theorem is almost an 'iff' statement, thus the assumptions on $L, H$ are the "weakest possible".

\subsubsection{The case of abelian groups}

One often needs results of such type for abelian groups, where one solves the equation $L(y-v)+H(y)=0$. We state the corresponding criterion separately.

Corollary 3.3 (i) Given abelian groups $V, W$ and a homomorphism $V \stackrel{L}{\longrightarrow} W$. Suppose there exists a decreasing filtration $V_{\bullet}$ of $V$ such that for all $y \in V_{1}$ and $j \geqslant 1$, $H\left(y+V_{j}\right)-H(y) \subseteq L\left(V_{j+1}\right)$. Then for any $v \in V_{1}$ there exists a quasi-good order-by-order solution.

(ii) If $V$ is complete with respect to $V_{\bullet}$ then the conditions $\left\{H\left(y+V_{j}\right)-H(y) \subseteq\right.$ $\left.L\left(V_{j+1}\right)\right\}_{j \geqslant 1}$ imply a quasi-good solution of the equation $L(y-v)+H(y)=0$. 
Remark 3.4 In the classical case of the equation $F(x, y)=0$ one requires that the map $V \stackrel{F_{y}^{\prime}(x, 0)}{\longrightarrow} W$ is right invertible, i.e. surjective. Our criterion demands that $F_{y}^{\prime}(x, 0)\left(V_{j+1}\right)$ contains the variation of the higher order terms $H\left(y+V_{j}\right)-H(y)$, here $H(y)=F(x, y)-F(x, 0)-F_{y}^{\prime}(x, 0) y$.

\subsubsection{A special version for group-action equations}

Given two maps of (not necessarily abelian) groups $V \underset{F}{\stackrel{L}{\rightrightarrows}} W$. Suppose $W$ is filtered by normal subgroups $\left\{W_{j}\right\}$ and $F(V)$ contains $\mathbb{1}_{W} \in W$. (Here we do not assume that $L$ is a homomorphism.) Denote by $\overline{L(V)}, \overline{F(V)} \subseteq W$ the closures with respect to the filtration $W_{i}$. The following statement is almost tautological, yet highly useful in Sect. 6.

Lemma 3.5 Suppose $F(V) \cdot\left(L(V) \cap W_{j}\right) \subseteq F(V) \cdot W_{j+1}$ for any $j \geqslant k$. If $W_{k} \subset$ $\overline{L(V)}$ then $W_{k} \subseteq \overline{F(V)}$.

In the abelian case the condition reads $F(V)+\left(L(V) \cap W_{j}\right) \subseteq F(V)+W_{j+1}$.

Proof Suppose $W_{k} \subseteq L(V)$, then $W_{j} \subseteq L(V)$ for any $j \geqslant k$. Thus $F(V) \cdot(L(V) \cap$ $\left.W_{k}\right) \subseteq F(V) \cdot W_{k+1} \subseteq \cdots$. As $F(V)$ contains $\mathbb{1}_{W} \in W$ we get $W_{k} \subseteq F(V) \cdot W_{N}$ for any $N$. Which is precisely $W_{k} \subseteq \overline{F(V)}$.

The general case. Let $N>k$, consider the quotient $W \stackrel{\pi_{N}}{\longrightarrow} W / W_{N}$. Denote the composition maps $V \stackrel{L}{\vec{F}} W \stackrel{\pi_{N}}{\longrightarrow} W / W_{N}$ by $\pi_{N} L, \pi_{N} F$. Then $W_{k} \subset \overline{L(V)}$ implies $\pi_{N}\left(W_{k}\right) \subseteq \pi_{N} L(V)$ for any $N$. By the previous paragraph we get $\pi_{N}\left(W_{k}\right) \subseteq$ $\pi_{N} F(V)$. Thus $W_{k} \subseteq F(V) \cdot W_{N}$ for any $N$, which means $W_{k} \subseteq \overline{F(V)}$.

\subsection{Criteria for modules over the rings}

Theorem 2.1 and Corollary 3.3 transform the solvability question into the search for an appropriate filtration $V_{\bullet}$. Not much can be said for a general (non)abelian group. However, our criterion simplifies for modules over a ring: it is enough to find just the first submodule $V_{1} \subset V$ and an ideal.

Let $R$ be a (commutative, associative) ring over a domain $\mathbb{k}$ of zero characteristic (e.g. $\mathbb{k}$ is a field). Given two $R$-modules and a homomorphism $L \in \operatorname{Hom}_{R}(V, W)$. Suppose further that the term $H(y)$ admits a "linear approximation with the remainder in the form of Lagrange", i.e.

$$
H(y+\Delta)=H(y)+H_{1}(y)(\Delta)+H_{2}(y, \Delta)(\Delta, \Delta),
$$

here $H_{1}(y)(z)$ is linear in $z$ while $H_{2}(y, \Delta)(z, z)$ is quadratic in $z$.

Example 3.6 Such an approximation holds e.g. for $R$ a subring of one of the quotients $\mathbb{k}[[x]] / I, C^{\infty}\left(\mathbb{R}^{m}, 0\right) / I$.

Corollary 3.7 Fix some ideal $J \subset R$ and a submodule $V_{1} \subset V$. Under the assumptions as above we have: 
(a) If the equation $L(y-v)+H(y)=0$ admits a good order-by-order solution for the filtration $\left\{V_{i}=J^{i-1} V_{1}\right\}$ then $H\left(V_{1}\right) \subseteq J \cdot L\left(V_{1}\right)$.

(b) If $H\left(V_{1}\right) \subseteq J \cdot L\left(V_{1}\right)$ then $L(y-v)+H(y)=0$ admits a quasi-good order-byorder solution for the filtration $\left\{V_{i}=J^{i-1} V_{1}\right\}$. (If $L$ is right invertible then there exists a good order-by-order solution.)

Proof (a) By Theorem 2.1, the existence of a good solution implies $H\left(y+\Delta_{j}\right)-$ $H(y) \in J^{j} L\left(V_{1}\right)$ and hence $H\left(V_{1}\right) \subseteq J L\left(V_{1}\right)$.

(b) For any $t \in \mathbb{k}, \Delta \in V_{1}$ we have $H(y+t \Delta)-H(y) \in J L\left(V_{1}\right)$. Thus $t H_{1}(y)(\Delta)+$ $t^{2} H_{2}(y, t \Delta)(\Delta, \Delta) \in J L\left(V_{1}\right)$ for $t \in \mathbb{k}$. Then $H_{1}(y)(\Delta) \in J L\left(V_{1}\right)$ and $H_{2}(y, t \Delta)$ $(\Delta, \Delta) \in J L\left(V_{1}\right)$. Thus $H_{1}(y)\left(J^{k} \Delta\right) \subseteq J^{k+1} L\left(V_{1}\right)$ and $H_{2}(y, t \Delta)\left(J^{k} \Delta, J^{k} \Delta\right) \subseteq$ $J^{k+1} L\left(V_{1}\right)$. Thus $H\left(y+\Delta_{j}\right)-H(y) \in J^{j} L\left(V_{1}\right)$ is implied by $H\left(V_{1}\right) \subseteq J L\left(V_{1}\right)$. Now invoke Corollary 3.3 for the filtration $\left\{V_{i}=J^{i-1} V_{1}\right\}$.

Corollary 3.7 reduces the question (for modules over a ring) to the search for an appropriate submodule $V_{1} \subset V$. The simplest submodule is $V_{1}=J V$ for some ideal $J \subset R$.

Corollary 3.8 Suppose $H(y)$ has the linear approximation as in equation (4), and moreover $H(y)$ is of order $k \geqslant 2$, i.e. $H(J V) \subseteq J^{k} W$. If $J H(V) \subseteq J \cdot L(V)$ then there exists a quasi-good order-by-order solution $J \cdot V \stackrel{y}{\longrightarrow} J \cdot V$ with respect to the filtration $\left\{J^{i} V\right\}$.

(Proof: Apply Corollary 3.7 for the filtration $V_{i}=J^{i} V$.)

Example 3.9 Given the equation $L(y-v)+H(y)=0$, where $H$ is of order $k$ as above.

(a) Consider the annihilator of cokernel ideal $\mathfrak{a}_{L}=\operatorname{ann}(\operatorname{coker}(L))$, cf. Sect. 2.3. If $J^{k} \subseteq J \cdot \mathfrak{m} \cdot \mathfrak{a}_{L}$ then there exists a good (order-by-order) solution $J \cdot V \stackrel{y}{\longrightarrow} J \cdot V$. Also, a bit weaker form: if $J^{k} \subseteq J \cdot \mathfrak{a}_{L}$ then there exists a good (order-by-order) solution $\mathfrak{m} \cdot J \cdot V \stackrel{y}{\longrightarrow} \mathfrak{m} \cdot J \cdot V$.

In the lowest order case, $k=2$, we get a sufficient condition for the order-byorder solvability: $J^{2} W \subseteq \mathfrak{m} J L(V)$. This condition is weaker than Tougeron's and Fisher's conditions, so even this criterion is stronger.

(b) Quite often $\mathfrak{m} \cdot L(V) \supseteq \mathfrak{a}_{L} \cdot W$, cf. Sect. 2.3. Then we get a stronger statement: if $J^{k} \subseteq J \cdot \mathfrak{a}_{L}$ then there exists a good (order-by-order) solution $J \cdot V \stackrel{y}{\longrightarrow} J \cdot V$.

\subsubsection{Ideals that satisfy $J^{2} \subseteq J \mathfrak{a}_{L}$}

(These are important in view of Example 3.9.) Consider the set $\mathfrak{J}$ of all ideals satisfying $J^{2} \subseteq J \mathfrak{a}_{L}$. This is an inductive set, i.e. for any increasing sequence $J_{1} \subseteq J_{2} \subseteq \ldots$ the union $\bigcup_{k} J_{k}$ is an ideal such that $J^{2} \subseteq J \mathfrak{a}_{L}$. (If $f, g \in \bigcup_{k} J_{k}$ then $f, g \in J_{k}$ for some $k<\infty$, thus $f g, f+g \in J_{k}$.) Therefore in $\mathfrak{J}$ there exist(s) ideal(s) that is/are maximal by inclusion.

Lemma 3.10 Let $J \subset R$ be a maximal by inclusion ideal that satisfies $J^{2} \subseteq J \mathfrak{a}_{L}$. 
(i) $\mathfrak{a}_{L} \subseteq J$. If the ideal $J$ is finitely generated then $J \subseteq \overline{\mathfrak{a}_{L}}$. (Here $\overline{\mathfrak{a}_{L}}$ is the integral closure.)

(ii) If $\mathfrak{a}_{L}$ is radical then $J=\mathfrak{a}_{L}$. If $R$ is integrallyclosed and $\mathfrak{a}_{L}$ is principal, generated by a non-zero divisor, then $J=\mathfrak{a}_{L}$.

Proof (i) If $J^{2} \subseteq J \mathfrak{a}_{L}$ then obviously the inclusion is satisfied by the ideal $J+\mathfrak{a}_{L}$ as well. As $J$ is the largest with this property, $\mathfrak{a}_{L} \subseteq J$. For the second part, note that $\mathfrak{a}_{L}$ is a reduction of $J$, see [17, Definition 1.2.1], thus $J \subseteq \overline{\mathfrak{a}_{L}}$ by [17, Corollary 1.2.5].

(ii) If $J^{2} \subseteq J \mathfrak{a}_{L}$ then in particular $J^{2} \subset \mathfrak{a}_{L}$. Then, $\mathfrak{a}_{L}$ being radical, we get $J \subseteq$ $\mathfrak{a}_{L}$. Hence together with (i) we get $J=\mathfrak{a}_{L}$. The second part follows from [17, Proposition 1.5.2]: in our case $\overline{\mathfrak{a}_{L}}=\mathfrak{a}_{L}$.

Example 3.11 In many cases $\mathfrak{a}_{L} \subsetneq J \subsetneq \overline{\mathfrak{a}_{L}}$ and a maximal by inclusion ideal $J$ is nonunique. For example, let $R=\mathbb{k}[[x, y, z]]$ and $L=\left(x^{p}, y^{p}, z^{p}\right) \in \operatorname{Mat}(1,3 ; R)$. Then $\mathfrak{a}_{L}=\left(x^{p}, y^{p}, z^{p}\right)$ while $\overline{\mathfrak{a}_{L}}=\mathfrak{m}^{p}$. Define $J_{z}=\left((x, y)^{p}, z^{p}\right), J_{y}=\left((x, z)^{p}, y^{p}\right)$, $J_{x}=\left((y, z)^{p}, x^{p}\right)$. By a direct check, each of them satisfies $J^{2}=J \mathfrak{a}_{L}$. But there is no bigger ideal $J$ that contains say $J_{x}+J_{y}$ and satisfies $J^{2}=J \mathfrak{a}_{L}$. Indeed, suppose $y^{p-i} z^{i} \in J$ and $x^{p-j} z^{j} \in J$, for some $i, j$ satisfying $i+j<p$. Then $J \mathfrak{a}_{L}=$ $J^{2} \ni x^{p-j} y^{p-i} z^{i+j}$, in particular $x^{p-j} y^{p-i} z^{i+j} \in \mathfrak{a}_{L}, i+j<p$, contradicting the definition of $\mathfrak{a}_{L}$. Thus, in this case there are at least three distinct maximal by inclusion ideals.

\section{3 (Non-)Uniqueness}

The classical Implicit Function Theorem ensures the uniqueness of solution, provided $F_{y}^{\prime}(0,0)$ is injective. In our case the injectivity ensures that the solution is "eventually unique" in the following sense.

Proposition 3.12 Given two order-by-order-solutions $y_{1}^{(n)}, y_{2}^{(n)}$ of the equation L $(y)$ $H(y)=L(v)$. Suppose $y_{1}^{(1)}, y_{2}^{(1)} \in V_{1}$ and $L$ is injective. Then for any $n, y_{1}^{(n)}\left(y_{2}^{(n)}\right)^{-1} \in$ $V_{n}$.

Proof By the assumption $y_{1}^{(1)}\left(y_{2}^{(1)}\right)^{-1} \in V_{1}$. Suppose the statement holds for $j=1$, $\ldots, n-1$. As both $y_{i}^{(n)}$ are Cauchy sequences, we get $y_{1}^{(n)}\left(y_{2}^{(n)}\right)^{-1} \in V_{n-1}$. We shall prove that in fact $y_{1}^{(n)}\left(y_{2}^{(n)}\right)^{-1} \in V_{n}$.

As each $y_{i}^{(n)}$ is an order-by-order-solution, we have $L\left(y_{i}^{(n)}\right) H\left(y_{i}^{(n)}\right) \in L(v) V_{n}$. Thus

$$
\left(L\left(y_{2}^{(n)}\right) H\left(y_{2}^{(n)}\right)\right)^{-1}\left(L\left(y_{1}^{(n)}\right) H\left(y_{1}^{(n)}\right)\right) \in V_{n} .
$$

By normality $V_{n} \triangleleft V$, we get

$$
\left(L\left(y_{2}^{(n)}\right)\right)^{-1} L\left(y_{1}^{(n)}\right) \in H\left(y_{2}^{(n)}\right)\left(H\left(y_{1}^{(n)}\right)\right)^{-1} V_{n} .
$$

Now use $y_{1}^{(n)}\left(y_{2}^{(n)}\right)^{-1} \in V_{n-1}$ and the property of higher order terms for $H$ to get $H\left(y_{2}^{(n)}\right)\left(H\left(y_{1}^{(n)}\right)\right)^{-1} \in L\left(V_{n}\right)$. Therefore, $L\left(\left(y_{2}^{(n)}\right)^{-1} y_{1}^{(n)}\right) \in L\left(V_{n}\right)$ and the statement follows by the injectivity of $L$. 
Remark 3.13 The assumption $y_{1}^{(1)}, y_{2}^{(1)} \in V_{1}$ is important. One might seek for a condition in terms of $v$ and $L$ only, then it is natural to ask whether $v$ belongs to a small enough subgroup of $V$. For example, in the case of modules, $v \in J V$, for some small enough ideal $J \subset R$. This does not suffice as one sees already in the example of one equation in one variable $\left(y-x^{a}\right)\left(y+x^{b}\right)=0$. Suppose $a<b$, then $\mathfrak{a}_{L}=\left(x^{a}\right)$, while $v \in\left(x^{a+b}\right)$. By taking $b \gg a$ the ideal $(x)^{a+b}$ can be made arbitrarily small as compared to $\mathfrak{a}_{L}$. Yet, there is no uniqueness.

Remark 3.14 If $L$ is non-injective then there can be no uniqueness. Even the images $L\left(y^{(n)}\right)$ of an order-by-order-solution are not "eventually unique". As the simplest example consider the equation $y_{1}^{2}+y_{2}^{2}-y_{1}+v=0$, where $v \in(x) \subset \mathbb{k}[[x]]$. We have a family of solutions $y_{1}=2\left(v+y_{2}^{2}\right) /\left(1+\sqrt{1-4\left(v+y_{2}^{2}\right)}\right)$, here $y_{2}$ is a parameter. By taking $y_{2} \in\left(v^{j}\right)$ these solutions can be made arbitrarily close one to the other (in particular they all lie in $\left.V_{1}\right)$, yet $L\left(y_{1}, y_{2}\right)$ is different for different $y_{2}$.

\subsection{A criterion for exact solutions}

The criteria of Sect. 3.1 provide order-by-order solutions, alternatively, solutions in the completion of $V$ by $V_{\bullet}$, i.e. the formal solutions. Recall the Artin approximation property: if a finite system of polynomial equations over $R$ has a solution over $\widehat{R}$ then it has a solution over $R[1,2]$. Many rings have this approximation property, e.g. excellent Henselian rings (in particular complete rings, analytic rings), cf. [16].

In our case we have more general rings and more general class of equations. Thus we give a criterion for exact (and not just order-by-order) solutions.

Fix some proper ideal $J \subsetneq R$. The pair $(R, J)$ is said to satisfy the (classical) Implicit Function Theorem, denote this by $\mathrm{cIFT}_{J}$, if for any surjective morphism of free $R$-modules of finite ranks $V \stackrel{L}{\longrightarrow} W$, any $v \in J V$ and any "higher order term" $V \stackrel{H}{\longrightarrow} W$, the equation $L(y-v)+H(y)=0$ has a good solution. Note that if $R$ satisfies cIFT $J$ then for any ideal $J_{1} \subseteq J$ the ring satisfies cIFT $_{J_{1}}$ as well.

Example 3.15 Let $(R, \mathfrak{m})$ be any local Henselian ring over a field $\mathbb{k}$. For example, the ring of formal power series $R=\mathbb{k}\left[\left[x_{1}, \ldots, x_{m}\right]\right] / I$, the ring of analytical power series $R=\mathbb{k}\left\{x_{1}, \ldots, x_{m}\right\} / I$ (for $\mathbb{k}$-normed), the ring of smooth functions $R=C^{\infty}\left(\mathbb{R}^{m}, 0\right) / I$ or the ring of $p$-times differentiable functions $R=C^{p}\left(\mathbb{R}^{m}, 0\right) / I$. Then $(R, \mathfrak{m})$ satisfies $\operatorname{cIFT}_{\mathfrak{m}}$.

The rings $\mathbb{k}[x], \mathbb{k}[x]_{(x)}$ do not satisfy $\operatorname{cIFT}_{\mathfrak{m}}$, e.g. the equation $y^{2}+y=x^{2}$ is not solvable over these rings.

We say that $(R, J)$ satisfies the Implicit Function Theorem with unit linear part, denote this by $\operatorname{IFT}_{J, \mathbb{1}}$, if the system of equations $y-v+H(y)=0$ has a good solution, $J V \stackrel{y}{\rightarrow} J V$, for any higher order terms $H$.

This system is a particular case of the classical implicit function equations. Therefore the Henselian rings (over a field) of Example 3.15 satisfy $\mathrm{IFT}_{\mathfrak{m} \mathbb{1}}$. Note that the condition $\mathrm{IFT}_{J, \mathbb{1}}$ is weaker than $\mathrm{IFT}_{J}$. For example, $\mathrm{IFT}_{J, \mathbb{1}}$ is satisfied by $\mathbb{Z}[[x]] / I$, $\mathbb{Z}\{x\} / I$ for $J=(x)$. More generally, one can take as $\mathbb{k}$ any ring and as $R$ a Henselian algebra over $\mathbb{k}$. 
Proposition 3.16 Given a finitely generated $R$-module $V$ and two maps $V \stackrel{L}{\vec{H}} W$.

Suppose $L \in \operatorname{Hom}_{R}(V, W)$, while $H$ satisfies $H\left(\sum_{i} y_{i} \xi_{i}\right)=\sum_{i} h_{i}\left(\left\{y_{j}\right\}\right) L\left(\xi_{i}\right)$, here $\left\{\xi_{i}\right\}$ are some generators of $V$, while $h_{i}\left(\left\{y_{j}\right\}\right)$ are of order $\geqslant 2$. Suppose IFT $_{J, 1}$ holds for an ideal $J \subsetneq R$. Then the equation $L(y-v)+H(y)=0$ has a solution $J V \stackrel{y}{\longrightarrow} J V$.

Note that here $R$ is not necessarily over a field, e.g. $R$ can be $\mathbb{Z}[[x]]$ or $\mathbb{Z}\{x\}$. Being of order $\geqslant 2$ means that $h_{i}(J) \subseteq J^{2} h(R)$ for any ideal $J \subseteq R$.

Proof Expand $v=\sum_{i} v_{i} \xi_{i}, y=\sum_{i} y_{i} \xi_{i}$, then the equation reads $\sum_{i}\left(y_{i}-v_{i}+\right.$ $\left.h_{i}(y)\right) L\left(\xi_{i}\right)=0$. Thus it is enough to solve the finite system of equations $\left\{y_{i}-v_{i}+\right.$ $\left.h_{i}(y)=0\right\}$. As $\operatorname{IFT}_{J, \mathbb{1}}$ holds in our situation we get the solution.

Corollary 3.17 Suppose a local ring $(R, \mathfrak{m})$ satisfies $\mathrm{IFT}_{\mathfrak{m} \mathbb{1}}$. Consider the equation $L(y-v)+H(y)=0$, where ord $H \geqslant 2$.

(i) If $J^{2} W \subseteq \mathfrak{m} J L(V)$ then for any $v \in J V$ there exists a solution.

(ii) If $J^{2} \subseteq J \mathfrak{a}_{L}$ then for any $v \in \mathfrak{m} J V$ there exists a solution.

(iii) If $J^{2} \subseteq J \mathfrak{a}_{L}$ and $\mathfrak{a}_{L} W \subseteq \mathfrak{m} L(V)$ then for any $v \in J V$ there exists a solution.

Example 3.18 Let $(R, \mathfrak{m})$ be a local Henselian ring over a field. Take $J=\mathfrak{a}_{L}$, then the corollary implies Tougeron's and Fisher's theorems. As mentioned in the introduction, if one takes $J$ the maximal possible that satisfies $J^{2}=J \mathfrak{a}_{L}$ then one gets the strengthening of Tougeron's and Fisher's theorems.

But the corollary is useful for more general rings, e.g. if in equation (2) the term $p\left(x_{1}, x_{2}\right)$ has integral coefficients then we get a solution over $\mathbb{Z}\left[\left[x_{1}, x_{2}\right]\right]$.

\section{Examples, remarks and applications}

\subsection{Comparison to Fisher's and Tougeron's theorems}

The condition $J^{2} \subseteq J \cdot \mathfrak{a}_{F_{y}^{\prime}(x, 0)}$ (cf. Corollary 3.17) is a weakening of the condition $J \subseteq \mathfrak{a}_{F_{y}^{\prime}(x, 0)}$.

Example 3.1 Let $R=\mathbb{k}\left[\left[x_{1}, x_{2}\right]\right]$, where $\mathbb{k}$ is some base ring, take $\mathfrak{m}=\left(x_{1}, x_{2}\right)$. (If $\mathbb{k}$ is a field then $\mathfrak{m}$ is the maximal ideal.) Consider the equation $H(y, x)+y_{1} x_{1}^{k}+y_{2} x_{2}^{k}+$ $p(x)=0$, compare this to (2). Here $H(y, x)$ represents the higher order terms, it is at least quadratic in $y_{1}, y_{2}$, while $p(x) \in R$. In this case, $L=F_{y}^{\prime}(x, 0)=\left(x_{1}^{k}, x_{2}^{k}\right) \in$ $\operatorname{Mat}(1,2 ; R)$ and $I_{\max }(L)=\mathfrak{a}_{L}=\left(x_{1}^{k}, x_{2}^{k}\right) \subset R$. Thus $\left(\mathfrak{a}_{L}\right)^{2}=\left(x_{1}^{2 k}, x_{1}^{k} x_{2}^{k}, x_{2}^{2 k}\right)$. Thus to apply Tougeron's and Fisher's theorems we have to assume that $p(x) \in$ $\mathfrak{m}\left(x_{1}^{2 k}, x_{1}^{k} x_{2}^{k}, x_{2}^{2 k}\right)$.

On the other hand, by a direct check, the ideal $\mathfrak{m}^{k}=\left(x_{1}, x_{2}\right)^{k}$ satisfies $\left(\mathfrak{m}^{k}\right)^{2}=$ $\mathfrak{m}^{k} \cdot\left(x_{1}^{k}, x_{2}^{k}\right)=\mathfrak{m}^{k} \cdot \mathfrak{a}_{L}$. Therefore Corollary 3.17 implies: if $p(x) \in \mathfrak{m}^{2 k+1}$ then the equation has a solution. For $\mathbb{k}$ an algebraically closed field we get a better criterion: if $p(x) \in \mathfrak{m}^{2 k}$ then the equation has a solution.

Note that to write down an explicit solution is not a trivial task even in the particular case of (2). 
Further, if $\mathbb{k}$ is not a field then we get the solvability of a "Diophantine type" equation. For example, for $\mathbb{k}=\mathbb{Z}$ and $H(y, x), p(x)$ defined over $\mathbb{Z}$, we get the criterion of solvability over $\mathbb{Z}[[x]]$. Note that even for the equation $y^{n}+y x^{k}+x^{N}=0$ the solvability over $\mathbb{Z}[[x]]$ is not totally obvious.

Therefore, even in the case of just one equation, the condition $J^{2}=J \cdot \mathfrak{a}_{L}$ strengthens the versions of Tougeron and Fisher.

\subsection{Comparison of the condition $J^{2}=J \cdot \mathfrak{a}_{\left.F_{y}^{\prime}(x, 0)\right)}$ to $H\left(V_{1}\right) \subseteq J L\left(V_{1}\right)$}

(cf. Corollary 3.7) It is simpler to check the ideals $J^{2}=J \cdot \mathfrak{a}_{\left.F_{y}^{\prime}(x, 0)\right)}$ than to look for a submodule satisfying the needed property. But the "ideal-type" criterion is in general weaker than the criterion via $V_{1}$.

Example 4.2 Consider the system

$$
\left\{\begin{array}{l}
y_{1}^{2}+y_{1} x_{1}=x_{1}^{n}, \\
y_{2}^{2}+y_{2} x_{2}=x_{2}^{m}
\end{array}\right.
$$

over $R=\mathbb{k}\left[\left[x_{1}, x_{2}\right]\right]$. In this case the annihilator of cokernel ideal is principal, $\mathfrak{a}_{F_{y}^{\prime}(x, 0)}=\left(x_{1} x_{2}\right)$, thus $J^{2}=J \cdot \mathfrak{a}_{F_{y}^{\prime}(x, 0)}$ implies $J=\mathfrak{a}_{F_{y}^{\prime}(x, 0)}$, see Sect. 3.2.1. And $\left(x_{1} x_{2}\right)$ does not contain $x_{1}^{n}, x_{2}^{m}$ regardless of how big $n$ and $m$ are.

Of course, the general criterion of Corollary 3.7 suffices here. (One starts from $V_{1}=\left(\begin{array}{l}x_{1} R \\ x_{2} R\end{array}\right)$ and $J=\left(x_{1}, x_{2}\right)$.)

This is a good place to see in a nutshell why no weakening of $J^{2}=J \cdot \mathfrak{a}_{F_{y}^{\prime}(x, 0)}$ in the form of some condition on ideals is possible.

Example 4.3 Consider the related system with a modified quadratic part

$$
\left\{\begin{array}{l}
y_{1}^{2}+y_{1} x_{1}=x_{1}^{n}, \\
y_{1}^{2}+y_{2} x_{2}=x_{2}^{m} .
\end{array}\right.
$$

While the previous system has obvious solutions for $n, m \geqslant 2$, this system has no solutions in $R$. Indeed, from the second equation it follows that $y_{1}$ is divisible by $x_{2}$. Then the left hand side of the first equation must be divisible as well, contradicting the non-divisibility of the right hand side.

Example 4.4 As a further illustration we consider the system

$$
\left\{\begin{array}{l}
y_{2}^{2}+y_{1} a_{1}=b_{1}, \\
y_{1}^{2}+y_{2} a_{2}=b_{2},
\end{array}\right.
$$

where $a_{i}, b_{i} \in \mathfrak{m} \subset R$, here $R$ is a regular local Henselian ring. Suppose $\operatorname{gcd}\left(a_{1}, a_{2}\right)=$ 1, i.e. $\left(a_{1}\right) \cap\left(a_{2}\right)=\left(a_{1} a_{2}\right)$. Then $\mathfrak{a}_{L}=\left(a_{1} a_{2}\right)$ is a principal ideal and thus $J^{2}=J \mathfrak{a}_{L}$ 
implies $J=\mathfrak{a}_{L}$. Hence the approach via $J^{2}=J \cdot \mathfrak{a}_{L}$ gives: if

$$
b_{1} \in \mathfrak{m}\left(a_{1}^{2} a_{2}\right), \quad b_{2} \in \mathfrak{m}\left(a_{1} a_{2}^{2}\right)
$$

then the system has a solution.

We check the approach via filtration. To invoke Corollary 3.7 we need $V_{1} \subset R^{\oplus 2}$ to satisfy the following condition: if $\left(v_{1}, v_{2}\right)^{\mathrm{T}}$ then

$$
\left(\begin{array}{l}
v_{2}^{2} \\
v_{1}^{2}
\end{array}\right) \in\left(\begin{array}{l}
a_{1} v_{1} \\
a_{2} v_{2}
\end{array}\right)
$$

for any $\left(v_{1}, v_{2}\right)^{\mathrm{T}}$ (here $\mathrm{T}$ stands for transposition). This gives: $V_{1} \subset\left(a_{1} a_{2}\right) R^{\oplus 2}$ and further substitution gives $V_{1} \subseteq\left(a_{1} a_{2}\right) R^{\oplus 2}$. Put $V_{1}=\left(a_{1} a_{2}\right) R^{\oplus 2}$, this ensures $H\left(V_{1}\right) \subset \mathfrak{m} L\left(V_{1}\right)$. Note that $L$ has the obvious continuous right inverse,

$$
\left(\begin{array}{l}
a_{1} h_{1} \\
a_{2} h_{2}
\end{array}\right) \stackrel{T}{\rightarrow}\left(\begin{array}{l}
h_{1} \\
h_{2}
\end{array}\right) .
$$

Thus for $b_{1} \in\left(a_{1}^{2} a_{2}\right), b_{2} \in\left(a_{1} a_{2}^{2}\right)$ the equation has a good order-by-order solution. The later condition is slightly weaker than (5).

Remark 4.5 Suppose the system of equations splits. Then it is natural to choose the split submodule: $V_{1}=V_{1,1} \oplus V_{1,2} \subset V$. (Note that the converse does not hold: decomposability of $V_{1}$ does not imply that the system splits in any sense. For example, all modules of the type $V_{1}=J V$ are decomposable if $V$ is free of rank $>1$.) The following questions are important:

- Suppose $L$ is block-diagonal. What are the conditions on $H$ so that we can choose $V_{1}=V_{1,1} \oplus V_{1,2}$ ?

- Formulate some similar statements for $L$ upper-block-triangular v.s. $V_{1}$ an appropriate extension of modules.

\subsection{Equations whose solutions are not good}

Often the "simple" and "most natural" solutions are not good (not even quasi-good) in our sense, moreover the (quasi-)good solutions do not exist at all.

Example 4.6 Consider the equation $y^{2}=p(x)$ over $R=\mathbb{k}[[x]]$. Here $L=0$, while $H(y) \neq 0$. Corollary 3.7 claims that there are no good solutions. Explicitly, there does not exist a submodule $\{0\} \neq V_{1} \subset R$ such that for any $p(x) \in V_{1}$ there exists a solution $V_{1} \stackrel{y}{\rightarrow} V_{1}$ good in the sense of Sect. 2.2. This can be seen directly, if $V_{1} \neq\{0\}$ then $x^{2 N+1} \in V_{1}$ for $N \gg 1$, and $y^{2}=x^{2 N+1}$ has no solutions in $R$.

Of course, by a direct check, for any $p(x)$ of even order there are solutions. But these solutions are not good. 
Example 4.7 Consider the equation $\left(y-g_{1}(x)\right)\left(y-g_{2}(x)\right)=0$ over $R=\mathbb{k}[[x]]$, $x=\left(x_{1}, \ldots, x_{n}\right), n>1$. Assume that $g_{1}(x), g_{2}(x)$ are generic enough, in particular $g_{1}(x) \cdot g_{2}(x) \notin\left(g_{1}(x)+g_{2}(x)\right)=L(V)$. Then the equation cannot be presented in the form $L(y-v)+H(y)=0$, so it has no quasi-good solutions. (Even its linear part is non-solvable, though the equation has two obvious solutions.) This happens because an arbitrarily small deformation of the free term, $g_{1}(x) g_{2}(x)$, will bring an equation with no solutions in $R=\mathbb{k}[[x]]$. (In the case $g_{1}(x), g_{2}(x) \in C^{\infty}\left(\mathbb{R}^{p}, 0\right.$ ) even a deformation by a flat function will lead to an equation with no solutions.)

\subsection{An application: bifurcations of polynomial roots}

Fix a polynomial $p(y)=\sum_{i=0}^{d} a_{i} y^{i}$. Suppose for a fixed tuple of the coefficients $\left(a_{0}, \ldots, a_{d}\right)$ the polynomial has only simple roots (of multiplicity one). Then under small deformations of coefficients the roots deform smoothly. The multiple roots cause bifurcations in general. Our results provide a sufficient condition that a particular root deforms (smoothly/analytically/etc.) under the change of parameters. More precisely, starting from the initial ring $R$ consider an extension $S$ of $R$ by one local variable, e.g. $S=R[[t]]$ or $S=R\{t\}$, etc. Present the family of equations in the form $a_{0}(t)+a_{1}(t) y+H(y, t)=0$. We say that a root $y_{0}$ of the initial equation deforms (smoothly/analytically/etc.) if there exists a root $y(t) \in S$ such that $y(0)=y_{0}$.

To formulate the criterion we shift the variables $y \mapsto y+y_{0}$, so that the (new) root of the initial equation is $y=0$.

Corollary 4.8 (i) (Tougeron) If $a_{0}(t) \in\left(t a_{1}^{2}(t)\right)$ then the root $y=0$ of the initial equation deforms with $t$.

(ii) (Belitskii-Kerner) If $a_{0}(t) \in\left(t a_{1}(t)\right)$ and $a_{i}(t)\left(a_{0}(t)\right)^{i-1} \in t\left(a_{1}(t)\right)^{i}$ for any $i \geqslant 2$ then the root $y=0$ of the initial equation deforms with $t$.

(Note that if $a_{0}(t) \in\left(t a_{1}^{2}(t)\right)$ then all assumptions of part two are satisfied.) To check this statement it is enough to put $v=a_{0} / a_{1}$ and $V_{j}=t^{j-1} \cdot(v)$.

Example 4.9 If all eigenvalues of a matrix are distinct, then they deform differentiably under small deformations of entries. In the case of multiple eigenvalues Corollary 4.8 ensures that at least one of potentially bifurcating eigenvalues deforms differentiably. More explicitly, expand the determinant

$$
\operatorname{det}\left(A_{t}-y \mathbb{1}\right)=\sum_{i \geqslant 2}(-y)^{i} \operatorname{trace} \wedge^{n-i} A_{t}=\operatorname{det} A_{t}-\left(\operatorname{trace} A_{t}^{\vee}\right) y+\cdots+(-y)^{n}
$$

(Here $\wedge^{n-i} A_{t}$ is the associated skew-power of $A_{t}$.) Suppose the multiple eigenvalue is zero, so det $A_{t=0}=0$. Then

- (Tougeron's part) If det $A_{t} \in\left(t\left(\text { trace } A_{t}^{\vee}\right)^{2}\right)$ then the eigenvalue deforms smoothly.

- (Belitskii-Kerners's part) If $\left(\operatorname{trace} \wedge^{n-i} A_{t}\right) \cdot\left(\operatorname{det} A_{t} / \operatorname{trace} A_{t}^{\vee}\right)^{i} \in\left(t \cdot \operatorname{det} A_{t}\right)$ then the eigenvalue deforms smoothly. 


\subsection{A possible application: smooth curve-germs on singular spaces}

Let $(X, 0) \subset\left(\mathbb{k}^{n}, 0\right)$ be a germ (algebraic/analytic/formal) of a singular space. The smooth curve-germs lying on $(X, 0)$ are an important subject, often used in the theory of arc spaces [8]. The first question is whether $(X, 0)$ admits at least one smooth curve-germ, [13-15].

From the IFT point of view this question reads (for simplicity we work over $\left.\mathbb{k}\left[\left[x_{1}, \ldots, x_{n}\right]\right]\right)$ : Can a given system of equations be augmented by another system so that the total system $\{F(x, y)=0=G(x, y)\}$ has a one-dimensional power series solutions? For example $x_{2}\left(x_{1}\right), \ldots, x_{n}\left(x_{1}\right) \in \mathbb{k}\left[\left[x_{1}\right]\right], F\left(x_{1}, x_{2}\left(x_{1}\right), \ldots, x_{n}\left(x_{1}\right)\right) \equiv$ 0 ? The strong IFT seems to lead to some results on the existence/properties of families of such curves.

\section{An approximation theorem of Artin-Tougeron type}

There are several approximation theorems guaranteeing analytic/ $C^{\infty}$-solutions, provided a formal solution exists. Given the germ of an analytic map at the origin $F:\left(\mathbb{R}^{m}, 0\right) \times\left(\mathbb{R}^{n}, 0\right) \rightarrow\left(\mathbb{R}^{p}, 0\right)$, consider the implicit function equation

$$
F(x, y)=0,
$$

here $x$ is the multi-variable, while $y$ is an unknown map, $\left(\mathbb{R}^{m}, 0\right) \stackrel{y}{\longrightarrow}\left(\mathbb{R}^{n}, 0\right)$.

A formal solution of this equation is a formal series $\widehat{y}(x) \in \mathbb{R}[[x]]^{\oplus n}$ such that $\widehat{F}(x, \widehat{y}(x)) \equiv 0$, where $\widehat{F}$ is the (formal) Taylor expansion at zero of the map $F$. In general this solution does not converge off the origin. Two classical results relate it to the "ordinary" solution.

Theorem 5.1 Let $\widehat{y}(x)$ be a formal solution of the analytic equation $F(x, y)=0$.

(i) For every $r \in \mathbb{N}$ there exists an analytic solution whose $r$ 'th jet coincides with the $r$ 'th jet of $\widehat{y}(x)[1]$.

(ii) There exists a $C^{\infty}$-solution $y(x)$ whose Taylor series at the origin is precisely $\widehat{y}(x)$ and such that for any $r \in \mathbb{N}$ there exists an analytic solution which is $r$-homotopic to $y(x)[31]$.

(Recall that two solutions $y_{0}(x), y_{1}(x)$ are $r$-homotopic if there exists a $C^{\infty}$-family of solutions $y(x, t)$ such that $y_{0}(x)=y(x, 0), y_{1}(x)=y(x, 1)$ and $y(x, t)-y_{0}(x)$ is $r$-flat at the origin.)

What if the equation $F(x, y)=0$ is not analytic but only of $C^{\infty}$-type? Does the existence of a formal solution for the completion $\widehat{F}(x, y)=0$ imply the existence of a $C^{\infty}$-solution? The naive generalization of Artin/Tougeron's theorems does not hold.

Example 5.2 Let $\tau$ be a function flat at the origin, e.g.

$$
\tau= \begin{cases}e^{-1 / x^{2}}, & x \neq 0 \\ 0, & x=0 .\end{cases}
$$


Consider the equation $\tau^{2}(x) y(x)=\tau(x)$. The completion of this equation is the identity $0 \equiv 0$ thus every formal series $\widehat{y} \in \mathbb{R}[[x]]$ is a formal solution of $\widehat{F}(x, y)=0$. However, the equation has no local smooth solutions (not even continuous ones).

In this example the coefficient of $y(x)$, i.e. the function $\tau^{2}$, is flat at zero. In other words, the ideal $\mathfrak{a}_{F_{y}^{\prime}\left(x, y_{0}\right)}$ is too small and $\mathfrak{a}_{F_{y}^{\prime}\left(x, y_{0}\right)} \cdot \mathfrak{m}^{\infty} \neq \mathfrak{m}^{\infty}$.

The following statement supplements our previous results and extends Tougeron's theorem to $C^{\infty}$-equations. Let $R=C^{\infty}\left(\mathbb{R}^{m}, 0\right)$ with the maximal ideal $\mathfrak{m} \subset R$. Suppose the equation $F(x, y)=0$ has a formal solution $\widehat{y}_{0}$. By Borel's lemma [25] we can choose a $C^{\infty}$-map $y_{0}$ whose completion is $\widehat{y}_{0}$, thus $F\left(x, y_{0}\right)$ is a vector of flat functions.

Theorem 5.3 Suppose the equation $F(x, y)=0$ has a formal solution and there holds $\operatorname{det}\left(F_{y}^{\prime}\left(x, y_{0}\right) F_{y}^{\prime}\left(x, y_{0}\right)^{\mathrm{T}}\right) \mathfrak{m}^{\infty}=\mathfrak{m}^{\infty}$. Then there exists a $C^{\infty}$-map, $\left(\mathbb{R}^{m}, 0\right) \stackrel{y^{y}}{\longrightarrow}$ $\left(\mathbb{R}^{n}, 0\right)$ whose Taylor series at the origin is precisely $\widehat{y}_{0}$ and such that $F(x, y(x)) \equiv 0$.

Proof We seek the solution in the form $y=y_{0}+z$, where the map $z$ is flat. Expand $F\left(x, y_{0}+z\right)$ into the Taylor series with remainder

$$
F\left(x, y_{0}+z\right)=F\left(x, y_{0}\right)+F_{y}^{\prime}\left(x, y_{0}\right) z+\left(\int_{0}^{1}(1-t) \frac{\partial^{2} F\left(x, y_{0}+t z\right)}{\partial y^{2}} d t\right) z^{2} .
$$

Then the equation takes the form

$$
F_{y}^{\prime}\left(x, y_{0}\right) z+G(x, z)=-F\left(x, y_{0}\right)
$$

where the map $F\left(x, y_{0}\right)$ is flat. Note that the summand $G$ satisfies the condition $G(x, \lambda z)=\lambda^{2} h H(x, z, \lambda)$ with a $C^{\infty}$-map $H$ such that $H_{z}^{\prime}(x, 0, \lambda)=0$. We look for the solution of (7) in the form

$$
z=d(x) F_{y}^{\prime}\left(x, y_{0}\right)^{\mathrm{T}}\left(F_{y}^{\prime}\left(x, y_{0}\right) F_{y}^{\prime}\left(x, y_{0}\right)^{\mathrm{T}}\right)^{\vee} u,
$$

where $d(x)=\operatorname{det}\left(F_{y}^{\prime}\left(x, y_{0}\right) F_{y}^{\prime}\left(x, y_{0}\right)^{\mathrm{T}}\right)$ and $A^{\vee}$ denotes the adjugate matrix.

Then we arrive at the equation $d^{2}(x) u+d^{2}(x) \widetilde{G}(x, u)=-F\left(x, y_{0}\right)$ with the $C^{\infty}$-map $\widetilde{G}$ satisfying $\widetilde{G}_{u}^{\prime}(x, 0)=0$. Dividing by $d^{2}(x)$, we obtain the equation

$$
u+\widetilde{G}(x, u)=\tau(x),
$$

where the map $\tau$ is flat. By the classical Implicit Function Theorem, the latter equation has a local flat $C^{\infty}$-solution. Hence, the map $z$ satisfies (7) and $y=y_{0}+z$ is the solution we need.

Remark 5.4 (a) The assumption of the theorem can be stated as: Every function flat at the origin is divisible by $\operatorname{det}\left(F_{y}^{\prime}\left(x, y_{0}\right) F_{y}^{\prime}\left(x, y_{0}\right)^{\mathrm{T}}\right)$. In particular this implies that $F_{y}^{\prime}\left(x, y_{0}\right)$ is non-degenerate in some punctured neighborhood of the origin $0 \in\left(\mathbb{R}^{m}, 0\right)$. Note that $y_{0}$ is defined up to a flat function, but the assumption does not depend on this choice. 
(b) Recall that a function $g(x)$ is said to satisfy the Lojasiewicz condition (at the origin) if there exist constants $C>0$ and $\delta>0$ such that for any point $x \in\left(\mathbb{R}^{m}, 0\right)$ : $|g(x)| \geqslant C \operatorname{dist}(x, 0)^{\delta}$. As is proved, e.g. in [30, Section V.4], $g(x)$ satisfies the Lojasiewicz condition at the origin iff $g(x) \mathfrak{m}^{\infty}=\mathfrak{m}^{\infty}$. Thus the assumption of the theorem can be stated in the form

$$
\operatorname{det}\left(F_{y}^{\prime}\left(x, y_{0}\right) F_{y}^{\prime}\left(x, y_{0}\right)^{\mathrm{T}}\right) \geqslant C \operatorname{dist}(x, 0)^{\delta} \quad \text { for some } C, \delta>0
$$

(c) A similar statement can be proved for $C^{k}\left(\mathbb{R}^{p}, 0\right)$-functions, but then the solution is in general only in the $C^{k-2-\delta}$-class.

\section{Openness of group orbits and applications to the finite determinacy}

Given a module $W$ over some base ring $\mathbb{k}$ (we assume $\mathbb{k} \supseteq \mathbb{Q}$ ) with a decreasing filtration $\left\{W_{i}\right\}$. Consider the group of all $\mathbb{k}$-linear invertible maps that preserve the filtration $\mathrm{GL}\left(W_{\bullet}\right)=\left\{g \in \mathrm{GL}(W): g\left(W_{j}\right)=W_{j}\right\}$. Fix some subgroup $G \subseteq \mathrm{GL}\left(W_{\bullet}\right)$ and let $G^{0} \subseteq G$ be the unipotent subgroup, Sect. 6.1.1. Fix some element $w \in W$, consider the germ of its $G^{0}$-orbit $\left(G^{0} w, w\right)$ and the tangent space to this germ $T_{\left(G^{0} w, w\right)}$, Sect. 6.1.2. (Note that the existence of $T_{\left(G^{0} w, w\right)}$ places some restrictions on $G$, see (8).)

Theorem 6.1 If $W_{k} \subseteq \overline{T_{\left(G^{0} w, w\right)}}$ then $w+W_{k} \subseteq \overline{G^{0} w}$.

Here $\overline{(\cdots)}$ denotes the closure with respect to the filtration $W_{\bullet}$. Thus the statement is of the order-by-order-type. In particular, in the proof we can assume that $W$ is $W_{\bullet}-$ complete. The proof is given in Sect. 6.2, after some preparations in Sect. 6.1. Some immediate applications to the finite determinacy are given in Sect. 6.3.

\subsection{Preparations}

\subsubsection{The unipotent subgroup $G^{0}$}

Consider the system of projecting maps $W \stackrel{\pi_{j}}{\longrightarrow} W / W_{j}$. They induce projections on the group $\operatorname{GL}(W) \stackrel{\pi_{j}}{\longrightarrow} \mathrm{GL}\left(W / W_{j}\right)$ and accordingly the restrictions $G \stackrel{\pi_{j}}{\longrightarrow} \pi_{j}(G) \subseteq$ $\operatorname{GL}\left(W / W_{j}\right)$. (We use the same letter $\pi_{j}$, this causes no confusion.) We define the "unipotent" part of the group

$$
G^{0}=\left\{g \in G:\left.\pi_{j} g\right|_{W_{j-1 / W_{j}}}=\left.\mathrm{Id}\right|_{W_{j-1 / W_{j}}}, j>0\right\} .
$$

Example 6.2 Let $(R, \mathfrak{m})$ be a local ring as in Example 3.6.

(a) Let $G=\mathcal{R}$ be the group of local coordinate changes $x \mapsto \phi(x)$. They act on the elements of the ring by $f(x) \mapsto \phi^{*}(f(x))=f(\phi(x))$. For the filtration $\left\{\mathfrak{m}^{j}\right\}$ the group $G^{0}$ consists of the changes of the form $x \mapsto x+h(x)$, where $h(x) \in \mathfrak{m}^{2}$. 
(b) More generally, consider the group of automorphisms of a module $G=\operatorname{GL}(p, R)$ $\rtimes \mathcal{R} \circlearrowright R^{\oplus p}$ acting by $w(x) \mapsto U(x) w(\phi(x))$, where $\phi \in \mathcal{R}$, while $U(x)$ is an invertible matrix over $R$. Then

$$
\begin{aligned}
G^{0}=\{(U, \phi): \phi(x) & =x+h(x), h \in \mathfrak{m}^{2}, \\
U(x) & =\mathbb{1}+u(x), u(x) \in \operatorname{Mat}(p, p ; \mathfrak{m})\} .
\end{aligned}
$$

(c) Note that $G^{0}$ depends essentially on the filtration. In the previous examples we could take the filtration by the powers of some other ideal $\left\{J^{i}\right\}$ or just by a decreasing sequence of ideals.

\subsubsection{Logarithm, exponent and the tangent space}

As is mentioned after Theorem 6.1 we can pass to the completion of the module $\widehat{W}$ with respect to the $\left\{W_{j}\right\}$ filtration. Accordingly we have GL( $\left.\widehat{W}_{\bullet}\right) \supset \widehat{G} \supseteq \widehat{G}^{0}$, the completions of GL(W), $G, G^{0}$.

Among all $\mathbb{k}$-linear maps (not necessarily invertible) $\operatorname{End}_{\mathbb{k}}(\widehat{W})$ consider the nilpotent ones $\operatorname{End}_{\mathbb{k}}^{\text {nilp }}(\widehat{W})=\left\{\xi: \xi \widehat{W}_{j} \subseteq \widehat{W}_{j+1}\right\}$. Consider the logarithmic map (recall that $\mathbb{Q} \subset \mathbb{k})$

$$
\widehat{G}^{0} \stackrel{\ln }{\rightarrow} \operatorname{End}_{\mathbb{k}}^{\text {nilp }}(\widehat{W}), \quad g \mapsto \ln g=\sum_{k=1}^{\infty} \frac{(1-g)^{k}}{k} .
$$

As $g \in \widehat{G}^{0},(1-g) \widehat{W}_{j} \subseteq \widehat{W}_{j+1}$, thus the sum (though infinite) is a well-defined linear operator on $\widehat{W}$. As this logarithm is defined by the standard formula, we have $\ln a^{i} a^{j}=\ln a^{i}+\ln a^{j}$. But in general $\ln a b \neq \ln a+\ln b$, as $a, b$ do not commute. Nevertheless we assume that

$$
\text { the image } \ln \widehat{G}^{0} \text { is a } \mathbb{k} \text {-linear subspace of } \operatorname{End}_{\mathbb{k}}^{\text {nilp }}(\widehat{W}) \text {. }
$$

This is satisfied in many cases, e.g. in all our examples.

Definition 6.3 The tangent space to $\widehat{G}^{0}$ at the unit element is the $\mathbb{k}$-module $T_{\widehat{G}^{0}}=$ $\ln \widehat{G}^{0} \subseteq \operatorname{End}_{\mathbb{k}}(\widehat{W})$.

Consider the exponential map, $T_{\widehat{G}^{0}} \stackrel{\exp }{\longrightarrow} \mathrm{GL}^{0}\left(W_{\bullet}\right)$ defined by

$$
\exp \xi=\mathbb{1}+\sum_{k=1}^{\infty} \frac{\xi^{k}}{k !}
$$

As $\xi$ is a nilpotent endomorphism, the sum (though infinite) is a well-defined linear operator and is invertible.

Lemma 6.4 The image $\exp T_{\widehat{G}^{0}}=\widehat{G}^{0}$ and the maps $T_{\widehat{G}^{0}} \underset{\ln }{\stackrel{\exp }{\rightleftarrows}} \widehat{G}^{0}$ are mutual inverses.

Proof Let $\xi \in T_{\widehat{G}^{0}}$, then $\xi=\ln g$ for some $g \in \widehat{G}^{0}$. Thus exp $\xi=\exp \ln g=g \in \widehat{G}^{0}$. The maps $\ln$ and exp are mutual inverses as they are defined by the same Taylor series as the classical functions. 


\subsubsection{The relevant properties of the exponent and variation operator}

The $j$ 'th stabilizer of $w \in W$ is the subgroup $\operatorname{St}_{j}(w)=\left\{g \in G: \pi_{j}(g w)=\pi_{j}(w)\right\}$. For any $g \in G^{0}$ and $w \in W$ define the variation operator $\Delta_{w}(g)=g w-w$.

Lemma 6.5 The restriction $\left.\pi_{j+1} \Delta_{w}\right|_{\mathrm{St}_{j}(w) \cap G^{0}} \rightarrow \pi_{j+1} W_{j}, j \geqslant 1$, is a homomorphism of groups.

Proof First note that the image of $\left.\Delta_{w}\right|_{\mathrm{St}_{j}(w)}$ is indeed in $W_{j}$ as $\pi_{j}\left(\Delta_{w}(g)\right)=0$ for any $g \in \mathrm{St}_{j}(w)$. Let $g \in G^{0}$ and $h \in \mathrm{St}_{j}(w)$. Then

$$
\pi_{j+1}\left(\Delta_{w}(g h)\right)=\pi_{j+1}\left(\Delta_{h w}(g)+\Delta_{w}(h)\right)=\pi_{j+1}\left(\Delta_{w}(g)\right)+\pi_{j+1}\left(\Delta_{w}(h)\right),
$$

as $\pi_{j}(h w)=\pi_{j}(w)$.

Lemma 6.6 Let $\xi \in T_{\widehat{G}^{0}}$ and $w \in W$.

(i) $\pi_{j}(\exp \xi) \in \pi_{j}\left(\mathrm{St}_{j}(w)\right)$ iff $\pi_{j}(\xi w)=0 \in \pi_{j}(W)$.

(ii) If $\pi_{j}(\exp \xi) \in \pi_{j}\left(\operatorname{St}_{j}(w)\right)$ then $\pi_{j+1}\left(\Delta_{w}(\exp \xi)\right)=\pi_{j+1}(\xi w)$.

Proof (i) $(\Rightarrow)$ As the stabilizer is a group, $\pi_{j}(\exp t \xi w)=\pi_{j}(w)$ for all $t \in \mathbb{Z}$. The left hand side of this equation is a polynomial in $t$ because $\xi$ is nilpotent. As char $\mathbb{k}=0$ and $\mathbb{k} \supseteq \mathbb{Q}$, the equality holds for all $t \in \mathbb{k}$. But this implies $\pi_{j}(\xi w)=0$.

$(\Leftarrow)$ If $\pi_{j}(\xi w)=0$ then $\pi_{j}\left(\xi^{k} w\right)=0$, thus $\pi_{j}(\exp \xi) \in \pi_{j}\left(\operatorname{St}_{j}(w)\right)$.

(ii) The function $h(t)=\pi_{j}\left(\Delta_{w}(\exp t \xi)\right)$ is a polynomial in $t$. By Lemma 6.5, it is additive. Thus $h(t)=t c$ where $c=h(1)=\pi_{j}(\exp \xi w-w)=\pi_{j}(\xi w)$.

\subsection{Proof of Theorem 6.1}

As is explained after the statement of the theorem, it is enough to consider the completion $\widehat{G}^{0} \circlearrowright \widehat{W}$. Thus we can use the exponent map $T_{\widehat{G}^{0}} \stackrel{\exp }{\longrightarrow} \widehat{G}^{0}$, cf. Sect. 6.1.2. Fix some $w \in \widehat{W}$ and consider the corresponding maps

$$
T_{\widehat{G}^{0}} \underset{F(\lambda)=\exp \lambda w-w}{\stackrel{L(\lambda)=\lambda(w)}{\rightleftarrows}} \widehat{W} .
$$

Note that $L\left(T_{\widehat{G}^{0}}\right)=T_{\left(\widehat{G}^{0} w, w\right)}$ and $F\left(T_{\widehat{G}^{0}}\right)=\widehat{G}^{0} w-w$. Then the theorem can be formulated in the form: If $\widehat{W}_{k} \subseteq L\left(T_{\widehat{G}^{0}}\right)$ then $\widehat{W}_{k} \subseteq F\left(T_{\widehat{G}^{0}}\right)$.

Note that $F(0)=0 \in \widehat{W}$. The statement will follow from Lemma 3.5 if we show

$$
F\left(T_{\widehat{G}^{0}}\right)+\left(L\left(T_{\widehat{G}^{0}}\right) \cap \widehat{W}_{j}\right) \subseteq F\left(T_{\widehat{G}^{0}}\right)+\widehat{W}_{j+1}, \quad j \geqslant k .
$$

Thus we should check that for any $\mu, \lambda_{j} \in T_{\widehat{G}^{0}}$ such that $L\left(\lambda_{j}\right)=\lambda\left(w_{j}\right) \in \widehat{W}_{j}$ there exists $\mu^{\prime} \in T_{\widehat{G}^{0}}$ such that

$$
(\exp (\mu) w-w)+\lambda_{j}(w) \in\left(\exp \left(\mu^{\prime}\right) w-w\right)+\widehat{W}_{j+1} .
$$

Define $\mu^{\prime}$ by $\exp \mu^{\prime}=\exp \lambda_{j} \exp \mu$, by Lemma 6.4, such $\mu^{\prime}$ exists and is unique. Note that $\pi_{j}\left(\lambda_{j}(w)\right)=0$ for the chosen $w \in \widehat{W}$. Then, by Lemma 6.5, 


$$
\pi_{j+1}\left(\exp \left(\lambda_{j}\right) \exp (\mu) w-w\right)=\pi_{j+1}\left(\exp \left(\lambda_{j}\right) w-w\right)+\pi_{j+1}(\exp (\mu) w-w) .
$$

Further, as $\lambda_{j}(w) \in \widehat{W}_{j}$ we get by Lemma 6.6: $\pi_{j+1}\left(\exp \left(\lambda_{j}\right) w-w\right)=\pi_{j+1}\left(\lambda_{j}(w)\right)$. Altogether

$$
\exp \left(\lambda_{j}\right) \exp (\mu) w-w+\widehat{W}_{j+1}=\exp (\mu) w-w+\lambda_{j}(w)+\widehat{W}_{j+1},
$$

as needed.

\subsection{An application to finite determinacy}

Let $W$ be a module over a local ring $(R, \mathfrak{m})$ with the filtration $\left\{W_{j}=\mathfrak{m}^{j} W\right\}$. Suppose $G$ preserves the filtration, then if $\mathfrak{m}^{k} W \subseteq \overline{T_{\left(G^{0} w, w\right)}}$ we have

$$
w+\mathfrak{m}^{k} W \subseteq \overline{G^{0} w}
$$

When $R, W$ are complete this is a ready criterion, otherwise one uses the Artin approximation theorem (or Theorem 5.3 in the $C^{\infty}$-case). This recovers the classical criterion of [19-21], revised and generalized many times [7,11,32]: the determinacy is fixed on the tangent level.

Let $(R, \mathfrak{m})$ be as in Example 3.6. Below we describe several scenarios (the module and the group action), in each case it is enough to write down the corresponding tangent $\operatorname{space}(\mathrm{s})$.

Example 6.7 Let $W=R$, so one studies the determinacy of function germs. The group of local coordinate changes $x \mapsto x+\phi(x)$ acts by $f(x) \mapsto f(x+\phi(x))$ and induces the right equivalence $\mathcal{R}$. The contact equivalence $\mathcal{K}$ is induced by $f(x) \mapsto$ $(1+u(x)) f(x+\phi(x))$. The unipotent parts $\mathcal{R}^{0}, \mathcal{K}^{0}$ are realized for $u(x) \in \mathfrak{m}, \phi(x) \in$ $\mathfrak{m}^{2}$. Denote by $\operatorname{Der}\left(R, \mathfrak{m}^{2}\right)$ the $R$-module of all derivations from $R$ to $\mathfrak{m}^{2}$. The tangent space to the orbit is then $T_{\left(\mathcal{K}^{0} f, f\right)}=\operatorname{Der}\left(R, \mathfrak{m}^{2}\right)(f)+\mathfrak{m}(f)$. Thus we get, compare e.g. to [12, Theorem I.2.23]:

- If $\mathfrak{m}^{k} \subseteq \operatorname{Der}\left(R, \mathfrak{m}^{2}\right)(f)$ then $f$ is $k$ - $\mathcal{R}^{0}$-determined.

- If $\mathfrak{m}^{k} \subseteq \operatorname{Der}\left(R, \mathfrak{m}^{2}\right)(f)+\mathfrak{m}(f)$ then $f$ is $k$ - $\mathcal{K}^{0}$-determined.

Example 6.8 More generally, let $W=R^{\oplus p}$ with the filtration $\mathfrak{m}^{j} W$. The contact group action can be written as $f(x) \mapsto(\mathbb{1}+U) f(x+\phi(x))$, where $U \in \operatorname{GL}(p, R)$, $\phi \in \operatorname{Aut}(R)$. For the unipotent part $\mathcal{K}^{0}$ one has: $U \in \operatorname{Mat}(p, p ; \mathfrak{m}), \phi \in \mathfrak{m}^{2}$. Then $T_{\left(\mathcal{K}^{0} f, f\right)}=\operatorname{Der}\left(R, \mathfrak{m}^{2}\right) f+\operatorname{Mat}(p, p ; \mathfrak{m}) f$.

Example 6.9 Consider the $R$-module of matrices Mat $(m, n ; R)$. The groups $G_{\mathrm{lr}}=$ $\mathrm{GL}(m, R) \times \mathrm{GL}(n, R), \mathcal{R}, \mathcal{G}_{\mathrm{lr}}=G_{\mathrm{lr}} \rtimes \mathcal{R}$ act on $\operatorname{Mat}(m, n ; R)$ by $A(x) \rightarrow U A(\phi(x))$ $V^{-1}$. Then

$$
\begin{aligned}
& T_{\left(\mathcal{G}_{\mathrm{lr}}^{0} A, A\right)} \\
& \quad=\operatorname{Span}_{R}(U A-A V)_{(U, V) \in \operatorname{Mat}(m, m ; \mathfrak{m}) \times \operatorname{Mat}(n, n ; \mathfrak{m})}+\operatorname{Span}_{R}(\mathcal{D}(A))_{\mathcal{D} \in \operatorname{Der}\left(R, \mathfrak{m}^{2}\right)} .
\end{aligned}
$$


This group and various its subgroups are important in many areas. The determinacy questions are studied in $[4,5]$.

Example 6.10 When the hypersurface singularity $\{f=0\} \subset \operatorname{Spec}(R)$ is nonisolated, the tangent space $T_{\left(\mathcal{K}^{0} f, f\right)}$ does not contain $\mathfrak{m}^{k}$ for any $k$. Thus the filtration $\left\{\mathfrak{m}^{j}\right\}$ is irrelevant. It is natural to consider only the deformations preserving the singular locus. More precisely, for the ideal $\mathrm{Jac}_{f}+(f)$ consider the following saturation. Take the primary decomposition $\bigcap_{i} I_{i}$ and apply the procedure: if $\sqrt{I_{i}} \supsetneq \sqrt{I_{j}}$ then erase $I_{i}$ in this decomposition. Eventually one gets a saturated version $\left(\operatorname{Jac}_{f}+(f)\right)^{\text {sat }}$, geometrically this corresponds to removing the embedded components of lower dimension. Then one can consider either of the filtrations $W_{j}=\mathfrak{m}^{j-1}\left(\mathrm{Jac}_{f}+(f)\right)^{\text {sat }}$ or $W_{j}=\mathfrak{m}^{j-1} \int\left(\operatorname{Jac}_{f}+(f)\right)^{\text {sat }}$, here $\int I=\{g \in R: \operatorname{Der}(R)(g)+(g) \subseteq I\}$. The later filtration has been studied in [22,26,27].

In both cases one defines $\operatorname{Der}_{W_{1}}(R)=\left\{\mathcal{D} \in \operatorname{Der}\left(R, \mathfrak{m}^{2}\right): \mathcal{D} W_{1} \subseteq \mathfrak{m} W_{1}\right\}$ and considers the corresponding subgroup $\exp \left(\operatorname{Der}_{W_{1}}(R)\right)$ of $\mathcal{R}^{0}$. In both cases one has: If $\operatorname{Der}_{W_{1}}(R)(f) \supseteq \mathfrak{m}^{k} W_{1}$ then $f$ is $k$-determined for deformations inside $W_{1}$.

Acknowledgments We thank Jacek Bochnak, Herwig Hauser, Dorin Popescu, Jesús M. Ruiz, Eugenii Shustin, and Sergei Yakovenko for the attention and valuable suggestions. We also thank two referees, their numerous remarks have greatly improved the exposition.

\section{References}

1. Artin, M.: On the solutions of analytic equations. Invent. Math. 5, 277-291 (1968)

2. Artin, M.: Algebraic approximation of structures over complete local rings. Inst. Hautes Études Sci. Publ. Math. 36, 23-58 (1969)

3. Bandman, T., Garion, S., Kunyavskii, B.: Equations in simple matrix groups: algebra, geometry, arithmetic, dynamics. Cent. Eur. J. Math. 12(2), 175-211 (2014)

4. Belitski, G., Kerner, D.: Finite determinacy of matrices over local rings.I, arXiv:1212.6894

5. Belitski, G., Kerner, D.: Finite determinacy of matrices over local rings. II. Computation of ann $T_{(\Sigma, A)} / T_{(G A, A)}(2015)$. arXiv: 1501.07168

6. Bruschek, C., Hauser, H.: Arcs, cords, and felts-six instances of the linearization principle. Amer. J. Math. 132(4), 941-986 (2010)

7. Damon, J.: The Unfolding and Determinacy Theorems for Subgroups of $\mathscr{A}$ and $\mathscr{K}$. Mem. Amer. Math. Soc. 50 306, (1984)

8. Denef, J., Loeser, F.: Germs of arcs on singular algebraic varieties and motivic integration. Invent. Math. 135(1), 201-232 (1999)

9. Eisenbud, D.: Commutative algebra. With a view toward algebraic geometry. Graduate Texts in Mathematics, vol. 150. Springer-Verlag, New York (1995)

10. Fisher, B.: A note on Hensel's lemma in several variables. Proc. Amer. Math. Soc. 125(11), 3185-3189 (1997)

11. Gaffney, T.J.: Properties of Finitely Determined Germs. Thesis (Ph.D.) Brandeis University. 1976

12. Greuel, G.-M., Lossen, C., Shustin, E.: Introduction to singularities and deformations. Springer Monographs in Mathematics. Springer, Berlin, 2007. xii+471 pp

13. G. Gonzalez-Sprinberg, M. Lejeune-Jalabert, Courbes lisses sur les singularités de surface, C. R. Acad. Sci. Paris, t. Sétie, I, 318 (1994), pp. 653-656

14. Jiang, G., Oka, M.: Lines on non-degenerate surfaces. Kodaira's issue. Asian J. Math. 4(1), 97-113 (2000)

15. Jiang, G., Oka, M., Duc, T.P., Siersma, D.: Lines on Brieskorn-Pham surfaces. Kodai Math. J. 23(2), 214-223 (2000)

16. Rond, G., Hauser, H.: Artin Approximation, manuscript, http://homepage.univie.ac.at/herwig.hauser/ Publications/artin_survey_gr_2013.pdf 
17. Huneke, C., Swanson, I.: Integral closure of ideals, rings, and modules. London Mathematical Society Lecture Note Series, 336. Cambridge University Press, Cambridge, (2006)

18. Kurke, H., Pfister, G., Popescu, D., Roczen, M., Mostowski, T.: Die Approximationseigenschaft lokaler Ringe. Lecture Notes in Mathematics, Vol. 634. Springer-Verlag, Berlin-New York, (1978)

19. Mather, J.N.: Stability of $C^{\infty}$-mappings. I. The division theorem. Ann. of Math. (2) 87, 89-104 (1968)

20. Mather, J.N.: Stability of $C^{\infty}$-mappings. II. Infinitesimal stability implies stability. Ann. of Math. (2) 89, 254-291 (1969)

21. Mather, J.N.: Stability of $C^{\infty}$-mappings. III. Finitely determined map-germs. Inst. Hautes Études Sci. Publ. Math. 35, 279-308 (1968)

22. Pellikaan, R.: Finite determinacy of functions with nonisolated singularities. Proc. London Math. Soc. (3) 57(2), 357-382 (1988)

23. Rond, G.: Sur la linearite de la fonction de Artin. Ann. Sci. École Norm. Sup. (4) 38(6), 979-988 (2005)

24. Rond, G.: Bornes effectives des fonctions d'approximation des solutions formelles d'Équations binomiales. J. Algebra 323(9), 2547-2555 (2010)

25. Rudin, W.: Real and complex analysis. Third edition. McGraw-Hill Book Co., New York, 1987. xiv+416 $\mathrm{pp}$

26. Siersma, D.: Isolated line singularities. Singularities, Part 2 (Arcata, Calif., 1981), 485-496, Proc. Sympos. Pure Math., 40, Amer. Math. Soc., Providence, RI, 1983

27. Siersma, D.: Singularities with critical locus a 1-dimensional complete intersection and transversal type $A_{1}$. Topology Appl. 27(1), 51-73 (1987)

28. Tougeron, J.C.: Une généralisation du théorème des fonctions implicites. C. R. Acad. Sci. Paris Sér. A-B 262, A487-A489 (1966)

29. Tougeron, J.C.: Idéaux de fonctions différentiables. I. Ann. Inst. Fourier (Grenoble) 181968 fasc. 1, $177-240$

30. Tougeron, J.C.: Idéaux de fonctions différentiables. Ergebnisse der Mathematik und ihrer Grenzgebiete, Band 71. Springer-Verlag, Berlin-New York, (1972)

31. Tougeron, J.C.: Solutions d'un système d'équations analytiques réelles et applications. Ann. Inst. Fourier (Grenoble) 26 (1976), no. 3, x, 109-135

32. Wall, C.T.C.: Finite determinacy of smooth map-germs. Bull. London Math. Soc. 13(6), 481-539 (1981) 